\title{
G protein-coupled receptor kinase 2 promotes high-level Hedgehog signaling by regulating the active state of Smo through kinase-dependent and kinase-independent mechanisms in Drosophila
}

\author{
Yongbin Chen, ${ }^{1}$ Shuang Li, ${ }^{1}$ Chao Tong, ${ }^{1,5}$ Yun Zhao, ${ }^{1,6}$ Bing Wang, ${ }^{1}$ Yajuan Liu, ${ }^{2,3}$ Jianhang Jia, ${ }^{2,3}$ \\ and Jin Jiang ${ }^{1,4,7}$ \\ ${ }^{1}$ Department of Developmental Biology, University of Texas Southwestern Medical Center at Dallas, Dallas, Texas 75390, USA; \\ ${ }^{2}$ Markey Cancer Center, University of Kentucky, Lexington, Kentucky 40536, USA; ${ }^{3}$ Department of Molecular and Cellular \\ Biochemistry, University of Kentucky, Lexington, Kentucky 40536, USA; ${ }^{4}$ Department of Pharmacology, University of Texas \\ Southwestern Medical Center at Dallas, Dallas, Texas 75390, USA
}

G protein-coupled receptor kinase 2 (Gprk2/GRK2) plays a conserved role in modulating Hedgehog (Hh) pathway activity, but its mechanism of action remains unknown. Here we provide evidence that Gprk2 promotes high-level Hh signaling by regulating Smoothened (Smo) conformation through both kinase-dependent and kinase-independent mechanisms. Gprk2 promotes Smo activation by phosphorylating Smo C-terminal tail (C-tail) at Ser741/Thr742, which is facilitated by PKA and CK1 phosphorylation at adjacent Ser residues. In addition, Gprk2 forms a dimer/oligomer and binds Smo C-tail in a kinase activity-independent manner to stabilize the active Smo conformation, and promotes dimerization/oligomerization of Smo C-tail. Gprk2 expression is induced by $\mathrm{Hh}$ signaling, and Gprk2/Smo interaction is facilitated by PKA/CK1-mediated phosphorylation of Smo C-tail. Thus, Gprk2 forms a positive feedback loop and acts downstream from PKA and CK1 to facilitate high-level Hh signaling by promoting the active state of Smo through direct phosphorylation and molecular scaffolding.

[Keywords: Hedgehog; Smo; Gprk2/GRK2; GPCR; conformation; phosphorylation]

Supplemental material is available at http://www.genesdev.org.

Received May 16, 2010; revised version accepted August 4, 2010.

The Hedgehog (Hh) family of secreted proteins plays important roles during embryonic development and adult tissue homeostasis (Ingham and McMahon 2001; Jiang and Hui 2008; Varjosalo and Taipale 2008). Aberrant Hh signaling has been implicated in numerous human disorders, including birth defects and cancers (Villavicencio et al. 2000; Taipale and Beachy 2001). In embryonic development, Hh often functions as a morphogen to specify different cell fates in a concentration-dependent manner (Jiang and Hui 2008). For example, in Drosophila wing

Present addresses: ${ }^{5}$ Department of Molecular and Human Genetics, Baylor College of Medicine, Houston, TX 77030, USA; ${ }^{6}$ Shanghai Institute of Biochemistry and Cell Biology, Shanghai Institutes for Biological Science, Chinese Academy of Sciences, Shanghai, People's Republic of China. ${ }^{7}$ Corresponding author.

E-MAIL jin.jiang@utsouthwestern.edu; FAX (214) 648-1960.

Article is online at http://www.genesdev.org/cgi/doi/10.1101/gad.1948710. development, posterior ( $\mathrm{P}$ )-compartment cells express and secrete Hh proteins that move into the anterior (A) compartment to form a local concentration gradient that induces the expression of distinct Hh target genes at different concentrations. Low levels of Hh signaling suffice to activate decapentaplegic (dpp), whereas intermediate and high levels of Hh signaling activity are required to activate patched (ptc) and engrailed (en), respectively (Strigini and Cohen 1997; Ohlmeyer and Kalderon 1998; Methot and Basler 1999; Jia et al. 2004).

The reception system for the Hh signal consists of three transmembrane proteins: a 12-transmembrane protein, Patched (Ptc), as the Hh receptor; a single-span transmembrane protein, Ihog, as a coreceptor; and a seventransmembrane protein, Smoothened (Smo), as the obligatory Hh signal transducer (Jiang and Hui 2008; Varjosalo and Taipale 2008). Ptc inhibits Smo substoichiometrically 
through a poorly defined mechanism in the absence of $\mathrm{Hh}$ (Taipale et al. 2002). Hh interacts physically with Ptc and Ihog to alleviate Ptc inhibition of Smo (Chen and Struhl 1996; Stone et al. 1996; Casali and Struhl 2004; Zheng et al. 2010), allowing Smo to activate the latent $Z n$ finger transcription factor Cubitus interruptus (Ci)/Gli (Jiang and Hui 2008; Varjosalo and Taipale 2008).

In Drosophila wing development, Ci plays dual roles that are performed by two distinct forms (Aza-Blanc et al. 1997; Methot and Basler 1999). In the absence of Hh, the full-length $\mathrm{Ci}\left(\mathrm{Ci}^{\mathrm{F}}\right)$ undergoes extensive phosphorylation by PKA, GSK3, and CK1, which targets $\mathrm{Ci}^{\mathrm{F}}$ for $\mathrm{SCF}^{\text {Slimb }}$ mediated proteolytic processing to generate a truncated repressor form $\left(\mathrm{Ci}^{\mathrm{R}}\right)$ (Jia et al. 2002, 2005; Price and Kalderon 2002; Smelkinson et al. 2007). $\mathrm{Ci}^{\mathrm{F}}$ forms complexes with the Ser/Thr kinase Fused (Fu), the kinesinrelated protein Costal2 (Cos2), and Suppressor of fused (Sufu), which impedes $\mathrm{Ci}^{\mathrm{F}}$ nuclear translocation and blocks its activity in the nucleus (Robbins et al. 1997; Methot and Basler 2000; Wang et al. 2000; Wang and Holmgren 2000; Wang and Jiang 2004; Sisson et al. 2006). In addition, Cos2 facilitates Ci phosphorylation by recruiting PKA, GSK3, and CK1 (Zhang et al. 2005). In the presence of Hh, activated Smo dissociates or changes the composition of $\mathrm{Ci}-\mathrm{Cos} 2-\mathrm{kinase}$ complexes, thereby impeding $\mathrm{Ci}$ phosphorylation and processing (Zhang et al. 2005). Smo may also regulate $\mathrm{Ci}$ processing through Gai (Ogden et al. 2008). Furthermore, high levels of $\mathrm{Hh}$ convert $\mathrm{Ci}^{\mathrm{F}}$ into an active but labile form $\left(\mathrm{Ci}^{\mathrm{A}}\right)$ by alleviating Sufu-mediated repression through $\mathrm{Fu}$ (Ohlmeyer and Kalderon 1998).

How Smo is activated and how Smo transduces different levels of Hh signaling activity are still poorly understood. In Drosophila, Hh induces Smo phosphorylation at multiple sites in its C-terminal tail (C-tail) by PKA and CK1, which activates Smo by promoting both its cell surface accumulation and active conformation (Jia et al. 2004; Zhang et al. 2004; Apionishev et al. 2005; Zhao et al. 2007). In addition, the levels of Smo activity appear to correlate with its levels of phosphorylation (Jia et al. 2004; Zhao et al. 2007). In vertebrates, Sonic hedgehog (Shh) induces ciliary accumulation of Smo, which correlates with Shh pathway activation (Jiang and Hui 2008). In addition, Shh promotes Smo to adopt an active conformation similar to that of Drosophila Smo (dSmo), although the canonical PKA/CK1 phosphorylation sites found in dSmo are not present in mammalian Smo (Zhao et al. 2007).

Several studies have suggested that $G$ protein-coupled receptor (GPCR) kinase 2 (GRK2) promotes Shh signaling by regulating Smo (Chen et al. 2004; Meloni et al. 2006; Philipp et al. 2008), raising the possibility that GRK2 may substitute the role of PKA/CK1 to activate Smo in vertebrates. Interestingly, genetic studies in Drosophila suggest that Gprk2 also modulates Hh signaling (Molnar et al. 2007; Cheng et al. 2010). Nevertheless, the mechanism by which Gprk2/GRK2 regulates Hh signaling remains unknown in any system.

Although PKA and CK1 play an essential role in Smo activation in Drosophila, additional mechanisms may exist because the activity of a phosphomimetic Smo with all of the PKA/CK1 phosphorylation sites converted to
Asp was still up-regulated by Hh (Jia et al. 2004). In addition, Hh induces Smo phosphorylation at many additional sites by unidentified kinases (Zhang et al. 2004). To search for additional components that regulate Smo and Hh signaling, we carried out a genetic modifier screen and identified Gprk2 as a positive regulator of $\mathrm{Hh}$ signaling and essential for optimal Smo activation. We provide evidence that Gprk2 regulates Smo conformation and activity by directly phosphorylating Smo at S741/ T742, and this phosphorylation is regulated by PKA/CK1 phosphorylation at adjacent sites. Unexpectedly, we uncovered a kinase activity-independent mechanism for Gprk2 to regulate Hh signaling. We demonstrate that Gprk2 forms a dimer/oligomer and binds Smo C-tail in a manner regulated by PKA and CK1 phosphorylation to facilitate the dimerization/oligomerization of Smo C-tail, thereby promoting the active Smo conformation.

\section{Results}

Inactivation of Gprk2 enhances the defects caused by partial loss of Smo function

To identify additional Hh signaling components, we carried out a genetic modifier screen in which a collection of Exilexis deficiency lines were screened for modifications of a "fused wing" phenotype caused by overexpression of a dominant-negative form of Smo $\left(\mathrm{Smo}^{-\mathrm{PKA12}}\right)$ using wing-specific Gal4 drivers MS1096 or C765 (see the Materials and Methods). We identified more than a dozen deficiencies that enhanced the "fused wing" phenotype, one of which is $D f(3 R)$ Exel6219, which removes 10 genes, including the Drosophila Gprk2 (Fig. 1A-C). As mammalian GRK2 had been implicated in regulating Shh signaling (Chen et al. 2004), we proceeded to determine whether the modification was due to loss of Gprk2. We found that the heterozygote for Gprk2 ${ }^{06936}$, a P-element insertion allele of Gprk2 (Schneider and Spradling 1997), enhanced the phenotype caused by expressing Smo ${ }^{-P K A 12}$ with $C 765$ (C765-Smo-PKA12) (Fig. 1D). The modification by Gprk2 $2^{06936}$ is less severe compared with Gprk2 deficiency, likely due to its hypomorphic nature (Schneider and Spradling 1997). Indeed, several imprecise excision lines, including Gprk $2^{415}$, modified C765-Smo ${ }^{-P K A 12}$ to the same extent as Gprk2 deficiency (Fig. 1E). Consistent with Gprk2 ${ }^{415}$ being a strong or null allele, we found that Gprk2 protein expression was diminished in Gprk2 $2^{15}$ mutant clones (Supplemental Fig. S1A). Gprk $2^{415}$ homozygotes were viable when grown at $25^{\circ} \mathrm{C}$ but were semilethal at $29^{\circ} \mathrm{C}$, and escapers exhibited the "fused wing" phenotype (Supplmental Fig. S1B-D), which is indicative of partial loss of Hh signaling activity. We also generated transgenic RNAi lines targeting the Gprk2 coding sequence (Materials and Methods) and found that Gprk2 RNAi greatly enhanced the C765-Smo ${ }^{-P K A 12}$ phenotype (Fig. 1F). The modification of C765-Smo ${ }^{-P K A 12}$ by loss of Gprk2 is also reflected by changes in Hh target gene expression. For example, C765-Smo ${ }^{-P K A 12}$ caused a reduction in $p t c$ expression in A-compartment cells near the A/P boundary (Fig. 1H). Removal of one copy of Gprk2 


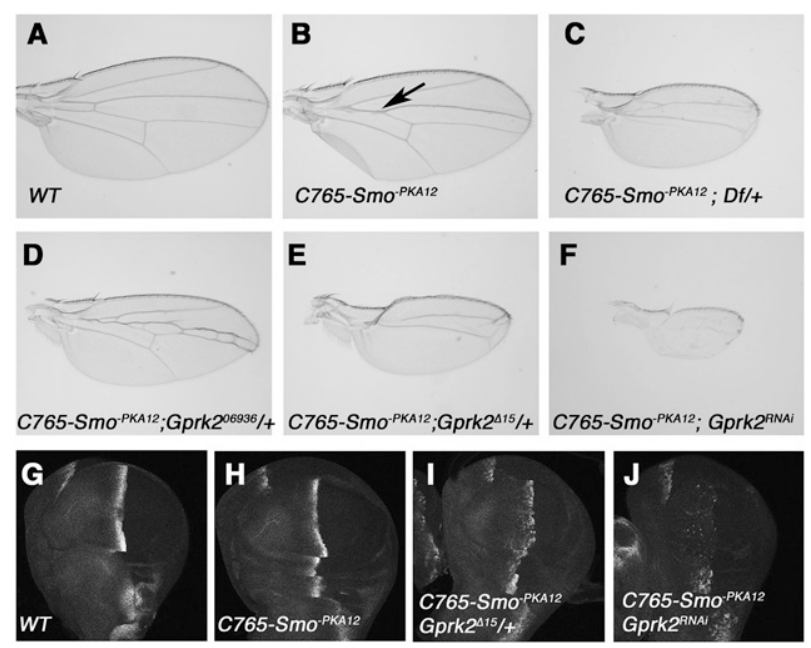

Figure 1. Reduction of Gprk2 modifies the phenotypes caused by a dominant-negative Smo. $(A, B)$ A wild-type wing $(A)$ or a wing expressing UAS-Smo ${ }^{-P K A 12}$ with the $C 765$ Gal4 driver (C765$\left.S m 0^{-P K A 12}\right)(B)$. (Arrow in $B$ ) Overexpression of $\mathrm{Smo}^{-\mathrm{PKA12}}$ causes a partial fusion between vein 3 and vein $4 .(C-F)$ Heterozygote for a Gprk2 deficiency $(C)$, a P-element insertion allele of Gprk2 $\left(G p r k 2^{06936}\right)(D)$, an excision allele $\left(G p r k 2^{415}\right)(E)$, or Gprk2 RNAi $(F)$ enhanced the fused wing phenotype caused by $C 765$ $S^{-P K A 12}$. $(G-J)$ C765-Smo ${ }^{-P K A 12}$ reduces ptc expression $(H)$, which is enhanced by the Gprk2 ${ }^{\Delta 15}$ heterozygote $(I)$ or Gprk2 RNAi $(J)$.

further reduced, whereas Gprk2 RNAi nearly abolished, ptc expression in C765-Smo ${ }^{-P K A 12}$ wing discs (Fig. 1I,J). Thus, reduction in Gprk2 activity exacerbates the Hh signaling defects caused by compromised Smo activity.

\section{Gprk2 is required for high-level Hh signaling}

To further investigate the role of Gprk2 in Hh signaling, we examined $\mathrm{Hh}$ target gene expression in wing discs in which Gprk2 is inactivated by either genetic mutations or RNAi. While levels of ptc and en expression were reduced in Gprk2 ${ }^{\Delta 15}$ homozygous mutant discs (Supplemental Fig. S2E-E",F-F"), $d p p$ was expressed at levels comparable with those in wild-type discs (Supplemental Fig. S2D-D"). In Gprk2 ${ }^{\Delta 15}$ mutant discs, $\mathrm{Ci}^{\mathrm{F}}$ was accumulated in A-compartment cells near the $\mathrm{A} / \mathrm{P}$ boundary similar to that in wild-type discs (indicated by arrows in Supplemental Fig. S2D-F), suggesting that loss of Gprk2 does not affect the low-level $\mathrm{Hh}$ signaling required for blocking $\mathrm{Ci}$ processing. However, $\mathrm{Ci}^{\mathrm{F}}$ was elevated in A-compartment cells immediately adjacent to the $\mathrm{A} / \mathrm{P}$ boundary (indicated by arrowheads in Supplemental Fig. S2D-F), implying that loss of Gprk2 prevents the conversion of $\mathrm{Ci}^{\mathrm{F}}$ into the labile $\mathrm{Ci}^{\mathrm{A}}$, a process requiring high-level Hh signaling/Ohlmeyer and Kalderon 1998). Gprk2 RNAi also reduced en expression and prevented the conversion of $\mathrm{Ci}^{\mathrm{F}}$ into the labile $\mathrm{Ci}^{\mathrm{A}}$ but did not affect $\mathrm{Ci}$ processing (Supplemental Fig. S3). These data are consistent with two recent studies (Molnar et al. 2007; Cheng et al. 2010). Thus, Gprk2 is required for high levels but not low levels of Hh signaling activity.

To determine whether Gprk2 is required in Hh-receiving cells to promote $\mathrm{Hh}$ signaling activity, we generated

Gprk2 ${ }^{415}$ mutant clones using the FRT/FLP-mediated mitotic recombination. We found that $G p r k 2^{\Delta 15}$ mutant clones near the A/P boundary exhibited reduced levels of ptc and en expression in a cell-autonomous fashion (Fig. 2D-D",F-F"), whereas P-compartment clones abutting the

A
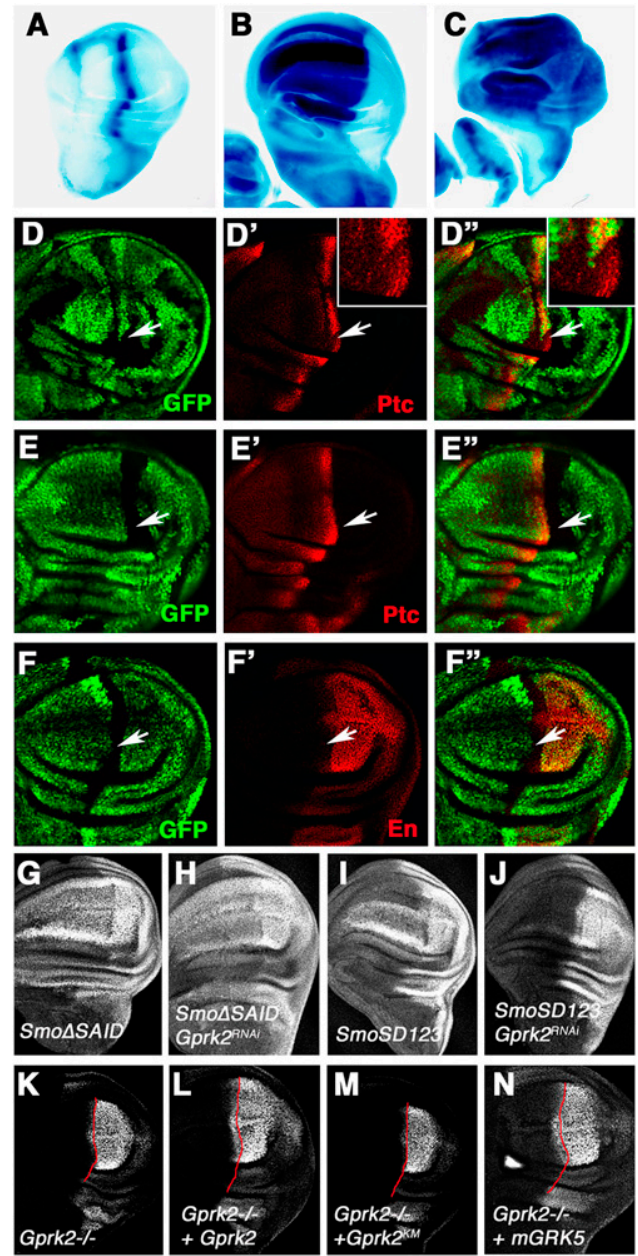

Figure 2. Genetic characterization of Gprk2. $(A-C)$ A Gprk2lac $Z$ enhancer trap is expressed along the A/P border $(A)$, and is induced ectopically by misexpression of either $\mathrm{Hh}(B)$ or an activated form of $\mathrm{Ci}(C)$ using MS1096 Gal4 driver. $\left(D-F^{\prime \prime}\right)$ A-compartment Gprk2 ${ }^{\Delta 15}$ mutant cells near the A/P boundary (marked by the lack of GFP) (arrows in $D-D^{\prime \prime}, F-F^{\prime \prime}$ ) exhibited reduced expression of ptc $\left(D^{\prime}\right)$ and en $\left(F^{\prime}\right)$. Insets in $\mathrm{D}^{\prime}$ and $\mathrm{D}^{\prime \prime}$ show enlarged images of the region indicated by the arrows. $\left(E-E^{\prime \prime}\right)$ P-compartment Gprk2 ${ }^{415}$ mutant cells abutting the $\mathrm{A} / \mathrm{P}$ boundary did not affect $p t c$ expression. $(G-J)$ Wing discs expressing Smo $\Delta$ SAID $(G, H)$ or SmoSD123 $(I, J)$ with $(H, J)$ or without $(G, I)$ multiple copies of a Gprk2 RNAi transgene using MS1096 were immunostained to show en expression. Smo $\Delta$ SAID but not SmoSD123 induced ectopic expression of en when Gprk2 was knocked down. $(K-N) G$ Grk $2^{415}$ mutant discs expressing a wildtype Gprk2 $(L)$, a kinase-dead Gprk2 $\left(\mathrm{Gprk} 2^{\mathrm{KM}}\right)(M)$, or a mammalian GRK5 (mGRK5) (N) with MS1096 were immunostained to show en expression. Expression of the wild-type Gprk2 or mGRK5 but not Gprk2 ${ }^{\mathrm{KM}}$ rescued anterior en expression in Gprk $2^{415}$ mutant discs. The red lines demarcate the A/P border based on Ci expression (not shown). 
A/P boundary did not affect Hh target gene expression (Fig. 2E-E"). Taken together, these observations suggest that Gprk2 acts in the Hh-receiving cells to promote high-level Hh signaling.

Gprk2 ${ }^{06936}$ harbors a lacZ reporter in the $5^{\prime}$ untranslated region (UTR) of the Gprk2 gene (Schneider and Spradling 1997). Both Gprk2-lacZ and Gprk2 protein are up-regulated along the A/P boundary of imaginal discs (Schneider and Spradling 1997), raising the possibility that Gprk2 transcription is regulated by $\mathrm{Hh}$ signaling. Indeed, Gprk2-lacZ was induced ectopically by misexpression of $\mathrm{Hh}$ or an activated form of Ci using the MS1096 Gal4 driver (Fig. 2AC; Molnar et al. 2007) and its expression was suppressed by Ptc overexpression (Molnar et al. 2007), suggesting that Gprk2 transcription is up-regulated by $\mathrm{Hh}$ signaling.

\section{Gprk2 regulates Smo activity}

In the GPCR signaling pathway, GRKs directly phosphorylate GPCRs in response to ligand stimulation, leading to desensitization of GPCR signaling (Pitcher et al. 1998). To determine how Gprk2 participates in Hh signaling, we determined the epistatic relationship between Gprk2 and Smo, which is structurally related to GPCRs. We examined two Smo variants: a phosphomimetic form (SmoSD123) with three clusters of PKA/CK1 sites converted to Asp (Jia et al. 2004); and Smo $\Delta 661-818$ (or Smo $\Delta$ SAID), in which the Smo autoinhibitory domain (SAID) was deleted (Zhao et al. 2007). Previous studies showed that both SmoSD123 and $\mathrm{Smo} \Delta \mathrm{SAID}$ exhibit constitutive Hh signaling activity and induce the ectopic expression of high-threshold Hhresponsive genes, including en, when overexpressed in wing discs (Fig. 2G,I; Jia et al. 2004; Zhao et al. 2007). Consistent with a previous study (Molnar et al. 2007), Gprk2 RNAi diminished the ectopic en expression and reduced the ectopic ptc expression induced by SmoSD123 (Fig. 2J; data not shown), suggesting Gprk2 is indispensable for the optimal activity of SmoSD123. In contrast, the activity of Smo $\Delta$ SAID was insensitive to loss of Gprk2, as Smo $\Delta$ SAID induced similar levels of ectopic ptc and $e n$ expression in Gprk2 RNAi discs (Fig. 2H; data not shown). These results suggest that Gprk2 acts at a step downstream from PKA/CK1 phosphorylation to counteract Smo autoinhibition.

\section{Gprk2 kinase activity is required for th signaling}

To determine if Gprk2 kinase activity is required for its function in the Hh pathway, we generated UAS transgenes expressing a wild-type or a kinase-dead form of Gprk2 (Gprk2 ${ }^{\mathrm{KM}}$ ) (see the Materials and Methods; below). Overexpression of UAS-Gprk2 but not UAS-Gprk2 $2^{K M}$ rescued anterior en expression in $G p r k 2^{\Delta 15}$ mutant discs (Fig. 2L,M), suggesting that the kinase activity of Gprk2 is essential for high-level Hh signaling. We also generated a UAS transgene expressing a mammalian GRK5 (mGRK5), which is highly homologous to Drosophila Gprk2. We found that expression of mGRK5 also rescued anterior en expression in Gprk2 ${ }^{\Delta 15}$ mutant discs (Fig. 2N), suggesting that mGRK5 can functionally substitute Drosophila Gprk2 to promote high-level Hh signaling activity.

\section{Gprk2 phosphorylates Smo C-tail}

To determine whether Gprk2 directly phosphorylates Smo, we applied an in vitro kinase assay in which GST fusion proteins containing various fragments of Smo C-tail were incubated with a recombinant human GRK5 in the presence of $\gamma_{-}{ }^{32}$ p-ATP. Initial mapping suggested that two regions of Smo C-tail (amino acids 661-818 and amino acids 889-1035) were phosphorylated by GRK5 (Fig. 3B, lanes 4,6). Further deletion analysis mapped the GRK sites to amino acids 700-748 and amino acids 997-1035 (Fig. 3B, lanes 12,18). GST-Smo661-731 was not phosphorylated by GRK5 (Fig. 3B, lane 13), suggesting that a GRK site is located between amino acids 731 and 748 that harbors the third PKA/CK1 phosphorylation cluster (Fig. 3A; Jia et al. 2004). A previous study showed that both $S_{741}$ and $T_{742}$ were phosphorylated in cl8 cells exposed to $\mathrm{Hh}$, but the corresponding kinase(s) remained unidentified (Zhang et al. 2004). As GRK family kinases tend to phosphorylate $\mathrm{S} / \mathrm{T}$ in an acidic environment (Premont et al. 1995), we speculated that $S_{741}$ and $T_{742}$ could be phosphorylated by Gprk2. Indeed, mutating $S_{741}$ and $\mathrm{T}_{742}$ in GST-Smo661-748 (GST-Smo661-748SATA) abolished GRK5-meidated phosphorylation in vitro (Fig. 3B, lane14). Similarly, mutating S1013 and S1015 in GSTSmo997-1035 (GST-Smo997-1035SA) abolished GRK5mediated phosphorylation of the corresponding Smo fragment (Fig. 3B, lane 19). These results suggest that $\mathrm{S}_{741} / \mathrm{T}_{742}$ and $\mathrm{S}_{1013} / \mathrm{S}_{1015}$ are GRK phosphorylation sites. We name $S_{741} / T_{742}$ collectively as GPS1 (GRK phosphorylation sites 1) and $S_{1013} / S_{1015}$ as GPS2 (Fig. 3A).

Because GPS1 is flanked by PKA (S740) and CK1 (S743 and S746) sites, phosphorylation of which improves the acidic environment (Fig. 3A), we tested whether phosphorylation at GPS1 could be enhanced by PKA/CK1 phosphorylation at adjacent sites. We constructed and purified GSTSmo700-748 fusion proteins with either wild-type GPS1 (WT) or GPS1 mutated to Ala (GPSA1), and carried out in vitro kinase assays using either recombinant GRK5 (Fig. 3C, lanes 1-8) or Flag-tagged Drosophila Gprk2 (FgGprk2) immunoprecipitated from S2 cells expressing FgGprk2 (Fig. 3C, lanes 9-16), with or without a prior phosphorylation by PKA and CK1 in the presence of cold ATP. We found that PKA/CK1 prior phosphorylation of GST-Smo700-748 WT enhanced its phosphorylation by GRK5 or FgGprk2 (Fig. 3C, cf. lanes 3,11 and 1,9). Mutating GPS1 to Ala in GSTSmo700-748 (GST-Smo700-748 ${ }^{\text {SATA) }}$ abolished both basal and PKA/CK1-stimulated phosphorylation by GRK (Fig. $3 C$, lanes $2,4,10,12)$. Substitution of the PKA site (S740A) or CK1 sites (S743 and 746A) to Ala abolished PKA/CK1stimulated GPS1 phosphorylation by GRK (Fig. 3C, lanes 5,6,13,14), whereas mutating PKA and CK1 sites to Asp (GST-Smo700-748 ${ }^{\mathrm{SD}}$ ) enhanced GPS1 phosphorylation in the absence of PKA and CK1 treatment (Fig. 3C, lanes $7,8,15,16)$. Taken together, these results demonstrate that PKA/CK1 phosphorylation at S740, S743, and S746 can stimulate GRK phosphorylation at GPS1. In contrast, we found that Gprk2 phosphorylation of GPS1 did not influence PKA/CK1 phosphorylation at adjacent sites (Supplemental Fig. S4). We also confirmed that the kinase-dead 

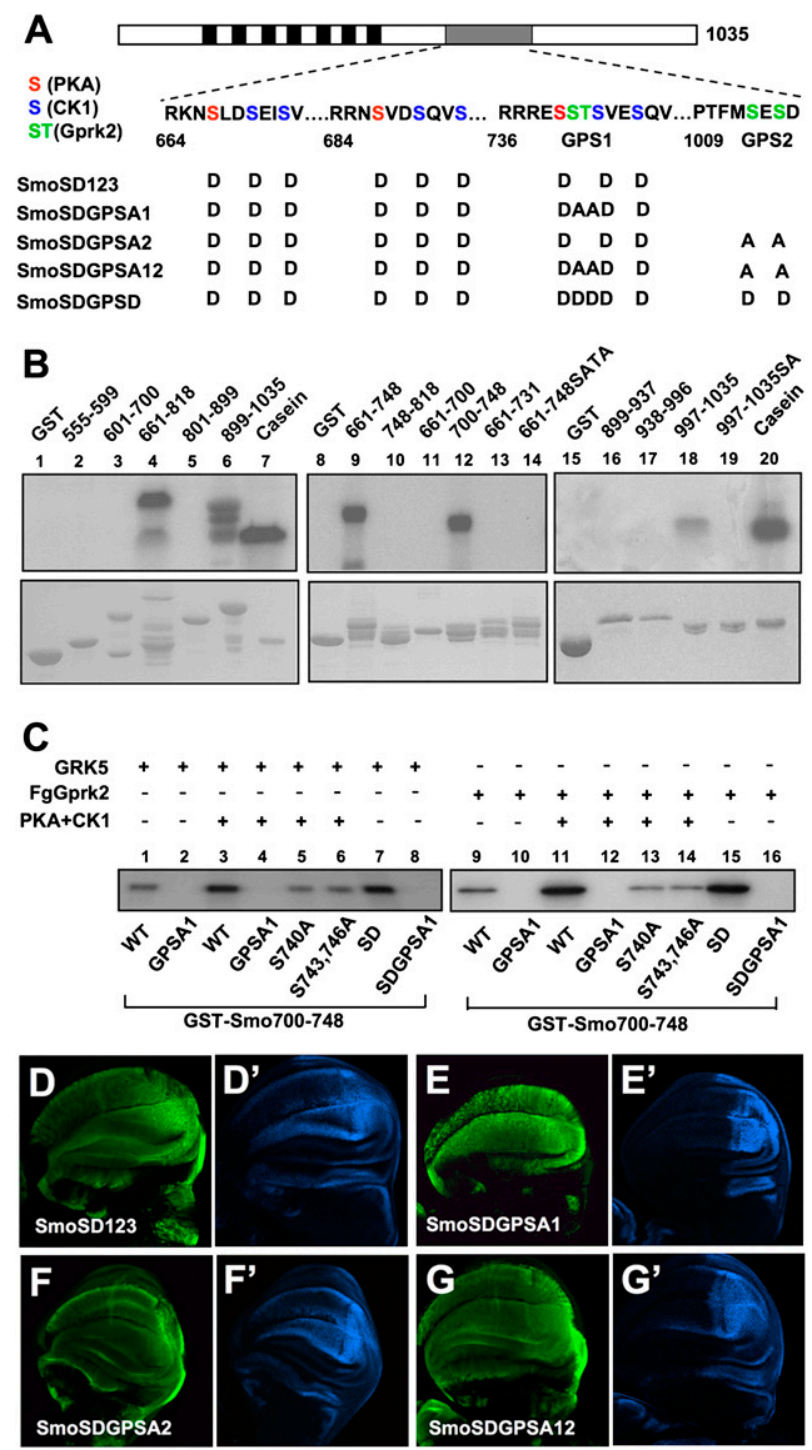

Figure 3. GRK phosphorylates multiple sites in Smo C-tail. (A) A schematic drawing of Smo with the sequence surrounding the PKA/CK1 sites and GRK sites (GPS1 and GPS2) shown below. PKA, CK1, and GRK sites are indicated by red, blue, and green residues, respectively. The transmembrane domains are indicated by the black boxes, and the SAID is indicated by the gray bar. Amino acid substitutions for SmoSD123 and its derivatives are listed. $(B)$ In vitro kinase assay using a recombinant GRK5 and GST fusion proteins carrying indicated fragments from the Smo C-tail. (Lanes 12,18) Two minimal fragments, amino acids 700748 and amino acids 997-1035, were phosphorylated by GRK5. (Lanes 14,19) Mutating the $\mathrm{S}_{741} / \mathrm{T}_{742}$ or $\mathrm{S}_{1013} / \mathrm{S}_{1015}$ abolished phosphorylation of the corresponding fragments. $(C)$ In vitro kinase assay using recombinant GRK5 (shown in lanes 1-8) or immunoprecipitated Fg-Gprk2 with GST-Smo700-748 bearing the wild-type sequence or indicated point mutations. PKA/ CK1 pretreatment was carried out in the presence of cold ATP. $\left(D-G^{\prime}\right)$ Wing discs expressing CFP-tagged SmoSD123 $\left(D, D^{\prime}\right)$, SmoSDGPSA1 $\left(E, E^{\prime}\right)$, SmoSDGPSA2 $\left(F, F^{\prime}\right)$, or SmoSDGPSA12 $\left(G, G^{\prime}\right)$ were immunostained to show the expression of CFP (green) and en (blue). SmoSD123 and SmoSDGPSA2 but not SmoSDGPSA1 or SmoSDGPSA12 induced ectopic en expression.
Gprk2 (Gprk2 $\left.{ }^{\mathrm{KM}}\right)$ did not phosphorylate GPS1 (Supplemental Fig. S5).

\section{In vivo function of GRK phosphorylation sites}

To determine the functional significance of Smo phosphorylation by Gprk2, we mutated GPS1 and GPS2 to Ala (SA) individually or in combination in the context of CFPtagged SmoSD123 to generate CFP-SmoSDGPSA1, CFPSmoSDGPSA2, and CFP-SmoSDGPSA12 (Fig. 3A). UAS transgenes expressing various Smo constructs were introduced into the 75B1 attP locus using the PhiC31 integration system to ensure the same level of transgene expression (Bischof et al. 2007). When expressed in wing discs using the MS1096 Gal4 driver, CFP-SmoSD123 induced ectopic en expression (Fig. 3D, $\mathrm{D}^{\prime}$ ). The ectopic en expression was diminished when GPS1 or both GPS1 and GPS2 were mutated to Ala (Fig. 3E, $E^{\prime}, G, G^{\prime}$ ). In contrast, CFP-SmoSDGPS2 still induced ectopic en expression (Fig. 3F, $\mathrm{F}^{\prime}$ ). The ectopic $p t c$ expression driven by SmoSD123 was slightly reduced by GPSA1 and GPSA12 mutations, but was not affected by GPSA2 mutation (data not shown). SmoSDGPSA1 and SmoSDGPSA12 still induced anterior overgrowth of wing discs (Fig. 3E, $\mathrm{E}^{\prime}, \mathrm{G}^{\prime}, \mathrm{G}^{\prime}$ ) and stabilized full-length Ci (data not shown). These results suggest that phosphorylation at GPS1 is required for maximal Smo activation.

We also mutated GPS1 and GPS2 to Asp to mimic phosphorylation in the context of CFP-SmoSD123 (CFPSmoSDGPSD) (Fig. 3A). CFP-SmoSDGPSD induced ectopic ptc and en expression at levels similar to those induced by CFP-SmoSD123 when expressed at high levels using the MS1096 Gal4 driver (data not shown). However, when expressed at lower levels using the $C 765$ Gal4 driver, CFP-SmoSDGPSD induced ectopic $p t c$ and en expression more robustly than CFP-SmoSD123 (Fig. 4A-D'), suggesting that phosphorylation at GPS1 increases SmoSD123 activity.

\section{Kinase-independent function of Gprk2 in Hh signaling}

If Gprk2 promotes Smo activation solely by phosphorylating Smo C-tail, we would expect that SmoSDGPSD should bypass the requirement of Gprk2 to induce highthreshold Hh responses. To our surprise, we found that SmoSDGPSD failed to induce ectopic en expression in Gprk2 mutant discs (Fig. 4F). One possibility is that Gprk2 may phosphorylate Smo at additional sites; for example, in the intracellular loop regions that were not examined by our in vitro kinase assay. Another possibility is that Gprk2 may phosphorylate other components of the Hh pathway acting downstream from Smo. However, the observation that Smo $\Delta$ SAID activity was insensitive to Gprk2 inactivation (Fig. 2G) does not favor these possibilities. A third possibility is that Gprk2 may promote Smo activity in a kinase-independent manner in addition to the phosphorylation-dependent mechanism. Indeed, kinase-independent desensitization of GPCR signaling by GRK2 was observed in cultured cells (Sallese et al. 2000). To test this latter possibility, SmoSDGPSD was coexpressed with the 

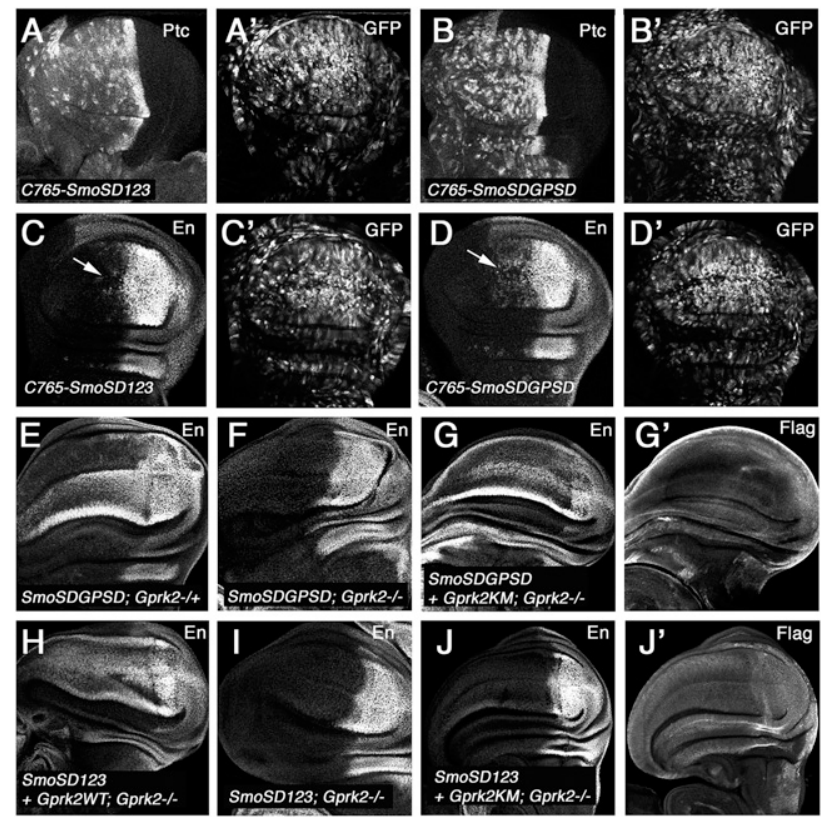

Figure 4. Gprk2 promotes Smo activation through phosphorylation-dependent and phosphorylation-independent mechanisms. $\left(A-D^{\prime}\right)$ Wing discs expressing UAS-SmoSD123-CFP $\left(A, A^{\prime}, C, C^{\prime}\right)$ or $U A S$-SmoSDGPSD-CFP $\left(B, B^{\prime}, D, D^{\prime}\right)$ with the $C 765$ Gal4 driver were immunostained to show the expression of ptc $(A, B)$, en $(C, D)$, and CFP $\left(A^{\prime}, B^{\prime}, C^{\prime}, D^{\prime}\right)$. $(E, F)$. en expression (visualized by anti-En antibody) in Gprk $2^{\Delta 15}$ heterozygous $(E)$ or homozygous $(F)$ wing discs expressing UAS-SmoSDGPSD-CFP with the MS1096 Gal4 driver. Loss of Gprk2 diminished the ectopic en expression induced by SmoSDGPSD. $\left(G, G^{\prime}\right)$ A $G p r k 2^{415}$ homozygous wing disc expressing SmoSDGPSD and Flag-tagged Gprk2 ${ }^{\mathrm{KM}}$ with MS1096 was immunostained with En and Flag antibodies. The kinase-dead Gprk2 rescued SmoSDGPSD-induced ectopic en expression in Gprk2 ${ }^{\Delta 15}$ mutant discs. $\left(H-J^{\prime}\right) G p r k 2^{\Delta 15}$ mutant discs expressing SmoSD123 alone $(I)$, or together with wild-type Gprk2 $(H)$ or Gprk2 ${ }^{\mathrm{KM}}\left(J, I^{\prime}\right)$ under the control of MS1096 were immunostained with anti-En and anti-Flag antibodies. Wild-type Gprk2 but not Gprk2 $2^{\mathrm{KM}}$ rescued SmoSD123-induced ectopic en expression in Gprk2 ${ }^{\Delta 15}$ mutant discs.

kinase-dead Gprk2 ${ }^{\mathrm{KM}}$ in Gprk2 mutant discs. We found that coexpression of Gprk2 ${ }^{\mathrm{KM}}$ restored the ectopic en expression induced by SmoSDGPSD in Gprk2 mutant discs (Fig. 4G). In contrast, when coexpressed in SmoSD123, only the wild-type Gprk2 (Gprk2 ${ }^{\mathrm{WT}}$ ) but not Gprk2 ${ }^{\mathrm{KM}}$ rescued the ectopic en expression in Gprk2 mutant discs (Fig. 4H-J). These results demonstrate that Gprk2 uses two paralleled mechanisms to promote high levels of Hh signaling activity: a kinase-dependent mechanism mainly through phosphorylation of $\mathrm{S}_{741} / \mathrm{T}_{742}$, and a kinase activity-independent mechanism.

\section{Gprk2 regulates Smo level}

Hh promotes Smo cell surface accumulation, which is mediated by PKA/CK1 phosphorylation (Denef et al. 2000; Zhu et al. 2003; Jia et al. 2004; Zhao et al. 2007). To determine whether Gprk2 regulates Smo activity by promoting its cell surface expression, we examined Smo expression in wing discs carrying Gprk2 mutant clones. To our surprise, we found that Smo protein levels were elevated in anterior Gprk2 clones, which is more evident in clones near the $\mathrm{A} / \mathrm{P}$ boundary where there are low levels of Hh (Fig. 5A, arrows; Molnar et al. 2007; Cheng et al. 2010). In contrast, loss of Gprk2 did not significantly affect Smo levels in A-compartment cells abutting the $\mathrm{A} / \mathrm{P}$ boundary or in P-compartment cells where Smo is normally accumulated in response to high levels of $\mathrm{Hh}$ (Fig. 5A, arrowheads; Molnar et al. 2007; Cheng et al.

A
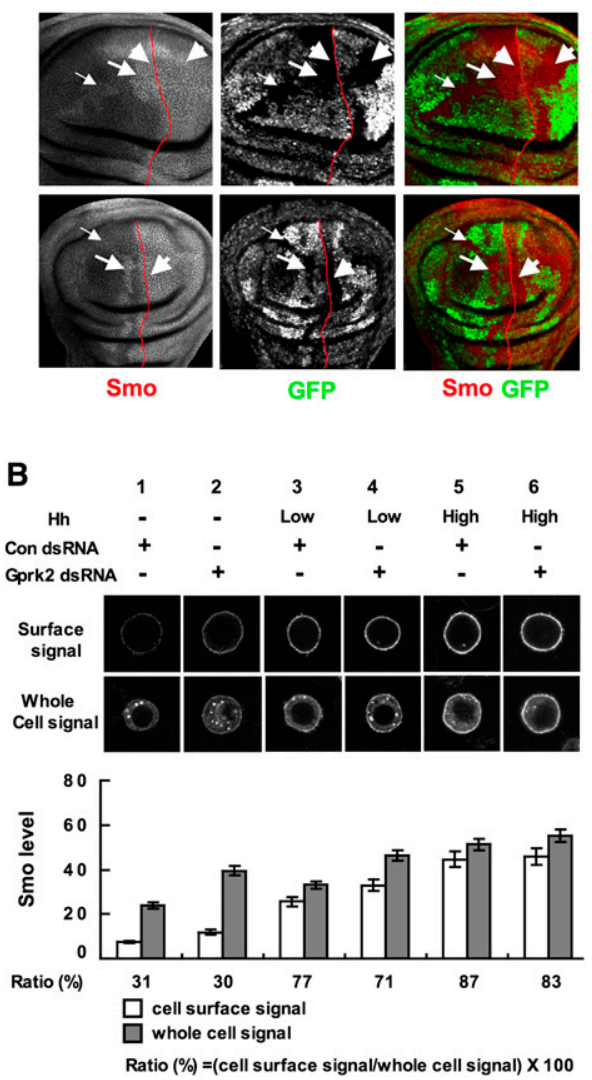

Figure 5. Gprk2 regulates Smo levels. $(A)$ Wing discs carrying Gprk2 $2^{415}$ mutant clones were immunostained to show the expression of Smo (red channel) and GFP (green channel). Gprk2 mutant cells are marked by the lack of GFP expression. Smo levels were elevated in anterior Gprk2 mutant clones located near (big arrows) or away from (small arrows) the A/P boundary, but not in anterior Gprk2 mutant cells immediately abutting the $\mathrm{A} / \mathrm{P}$ boundary or in $\mathrm{P}$-compartment cells (arrowheads). The A/P boundary is marked by red lines based on $\mathrm{Ci}$ costaining (not shown). (B) S2 cells stably expressing a Myc-tagged Smo under the control of metallothionein promoter were treated with Gprk2 dsRNA or control (Luciferase) dsRNA in the absence of Hh, or in the presence of low (one-tenth of high) or high levels of Hh. Cells were immunostained with anti-SmoN antibody before membrane permeabilization to visualize cell surface Smo (top panels), or after membrane permeabilization to examine the total Smo (bottom panels). Quantification of cell surface and total Smo levels are shown (mean $\pm \mathrm{SD} ; n \geq 20$ ). The numbers below the bars indicate the percentage of Smo on the cell surface. 
2010). Expressing wild-type Gprk2 but not Gprk2 ${ }^{\mathrm{KM}}$ in Gprk2 15 mutant wing discs suppressed Smo up-regulation in A-compartment cells away from the A/P boundary (Supplemental Fig. S6), suggesting that the kinase activity of Gprk2 is required for destabilization of Smo in these cells.

To determine whether loss of Gprk2 affects Smo subcellular localization, we turned to a cell-based assay using an S2 line that stably expressed a Myc-tagged Smo (MycSmo). Myc-Smo-expressing cells were treated with Gprk2 dsRNA or control dsRNA for $3 \mathrm{~d}$, followed by treatment with conditioned medium containing different levels of Hh-N for $24 \mathrm{~h}$. Cell surface or total Smo was visualized by immunostaining with anti-SmoN antibody prior to or after membrane permeabilization. As shown in Figure 5B, Gprk2 RNAi resulted in an increase in the levels of both cell surface and total Smo in the absence of $\mathrm{Hh}$ or in the presence of low levels of $\mathrm{Hh}$ (Fig. 5B, cf. lanes 2,4 and 1,3). In agreement with the in vivo results, inactivation of Gprk2 did not significantly alter the levels of either cell surface or total Smo in the presence of high levels of $\mathrm{Hh}$ (Fig. 5B, cf. lanes 6 and 5). Quantification analysis suggests that Gprk2 RNAi did not significantly increase the percentage of Smo on the cell surface compared with control RNAi (30\% vs. $31 \%$ ). In contrast, Hh treatment preferentially stabilized Smo on the cell surface as the percentage of Smo on the cell surface increased from 31\% to $77 \%$ and $87 \%$ in the presence of low and high levels of $\mathrm{Hh}$, respectively (Fig. 5B, lanes 3,5 ).

\section{Gprk2 promotes the active Smo conformation}

Our previous study demonstrated that Hh-induced phosphorylation of Smo triggers a conformational switch and dimerization of Smo C-tail, and that clustering of Smo C-tails promotes high-level Hh signaling activity (Zhao et al. 2007). The reduction of Smo activity in the absence of Gprk2 prompted us to investigate whether Gprk2 is required for Smo to adopt an active state. Clustering of Smo C-tails can be measured by fluorescence resonance energy transfer (FRET) analysis using a pair of $\mathrm{C}$ terminally CFP/YFP-tagged Smo constructs (Smo-CFP $/ \mathrm{YFP}^{\mathrm{C}}$ ) (Zhao et al. 2007). Accordingly, Smo-CFP ${ }^{\mathrm{C}}$ and Smo-YFP ${ }^{\mathrm{C}}$ were transfected into S2 cells treated with Gprk2 dsRNA or control dsRNA, followed by treatment with or without Hh-conditioned medium. FRET between Smo-CFPC and Smo-YFP ${ }^{\mathrm{C}}\left(\right.$ FRET $\left.^{\mathrm{C}}\right)$ was low in the absence of $\mathrm{Hh}$ but increased dramatically after Hh stimulation or when the PKA and CK1 sites were mutated to Asp to mimic phosphorylation (SmoSD123-CFPC/YFP ${ }^{\mathrm{C}}$ ) (Fig. 6A; Zhao et al. 2007). Gprk2 RNAi did not change the basal FRET ${ }^{\mathrm{C}}$ but significantly reduced the Hh-induced FRET ${ }^{\mathrm{C}}$ (Fig. 6A). In agreement with the observation that Gprk2 inactivation reduces the activity of SmoSD123 in wing discs (Fig. 2I), Gprk2 RNAi significantly reduced the FRET between SmoSD-CFP $/ \mathrm{YFP}^{\mathrm{C}}$ in S2 cells (Fig. 6A). We also found that Gprk2 RNAi affected the Hh-induced decrease in the intramolecular FRET between CFP inserted in the third intracellular loop and YFP fused to the C terminus of Smo
A

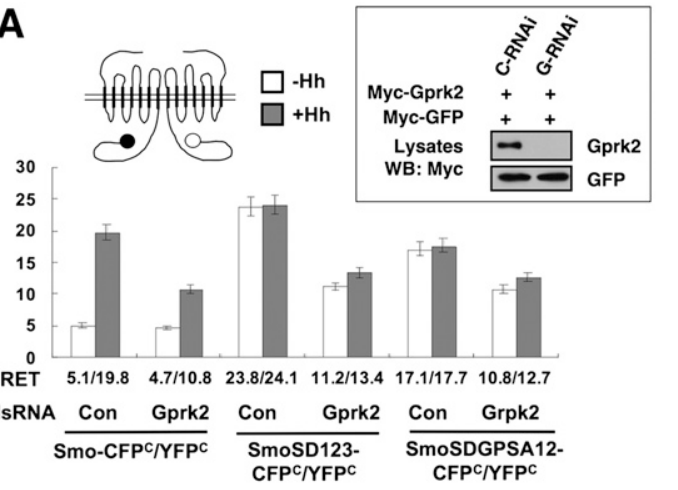

B
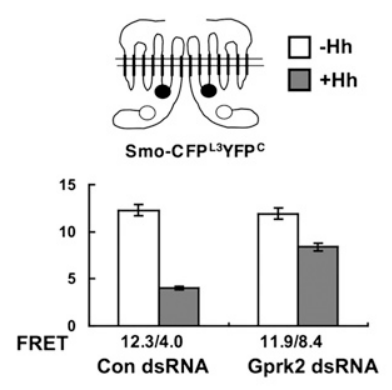

C
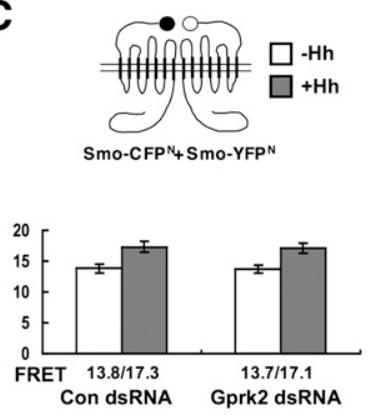

D
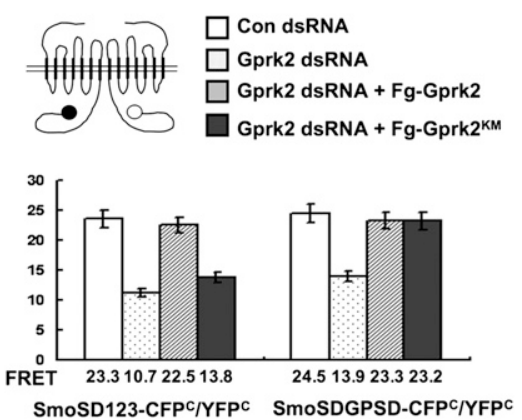

Figure 6. Gprk2 regulates Smo conformation. $(A-C)$ FRET efficiency from indicated wild-type or mutant $\mathrm{Smo-CFP} / \mathrm{YFP}^{\mathrm{C}}(A)$, Smo-CFP ${ }^{\mathrm{L} 3} \mathrm{YFP}^{\mathrm{C}}(B)$, or Smo-CFP $/ \mathrm{YFP}^{\mathrm{N}}(C)$ expressed in S2 treated with Gprk2 dsRNA or control (luciferase) dsRNA in the absence or presence of Hh treatment. Gprk2 RNAi reduced Hh-induced FRET from $\mathrm{Smo-CFP} /$ $\operatorname{YFP}^{\mathrm{C}}(A)$ and attenuated Hh-induced reduction in the FRET from Smo-CFP ${ }^{\mathrm{L} 3} \mathrm{YFP}^{\mathrm{C}}(B)$, but did not affect the FRET from Smo$\mathrm{CFP}^{\mathrm{N}} / \mathrm{YFP}^{\mathrm{N}}(C)$. (A) Gprk2 RNAi reduced both the basal and Hh-induced FRET from SmoSD123-CFP $/$ YFP $^{\mathrm{C}}$ or SmoSDGPSD$\mathrm{CFP}^{\mathrm{C}} / \mathrm{YFP}^{\mathrm{C}}$. Mean $\pm \mathrm{SD} ; n \geq 15$. The cartoons above the graphics indicate Smo biosensors, with filled and open circles representing CFP and YFP, respectively. The inset in $A$ shows that Gprk2 RNAi (G-RNAi) but not the control RNAi (C-RNAi) effectively knocked down transfected Myc-Gprk2 in S2 cells. Myc-GFP was cotransfected as a control for transgene expression and RNAi specificity. (D) S2 cells treated with Gprk2 or control dsRNA were transfected with SmoSD123-CFP ${ }^{\mathrm{C}} / \mathrm{YFP}^{\mathrm{C}}$ or SmoSDGPSD$\mathrm{CFP}^{\mathrm{C}} / \mathrm{YFP}^{\mathrm{C}}$ with or without cotransfection of Fg-Gprk2 or Fg-Gprk2 ${ }^{\mathrm{KM}}$ in the presence

of Hh-conditioned medium, followed by FRET analysis. Both the wild-type and kinase-dead Gprk2 restored high FRET from SmoSDGPSD-CFP ${ }^{\mathrm{C}} / \mathrm{YFP}^{\mathrm{C}}$ after endogenous Gprk2 was depleted by Gprk2 RNAi. In contrast, only the wild-type but not the kinasedead Gprk2 rescued the FRET from SmoSD123-CFPC/YFP ${ }^{\mathrm{C}}$ after Gprk2 RNAi. Mean \pm SD; $n \geq 15$. 
(SmoCFP ${ }^{\mathrm{L} 3} \mathrm{YFP}^{\mathrm{C}}$ ) (Fig. 6B). In contrast, Gprk2 RNAi did not affect the FRET between the $\mathrm{N}$ terminally CFP/YFPtagged Smo constructs $\left(\right.$ Smo-CFP ${ }^{\mathrm{N}} / \mathrm{YFP}^{\mathrm{N}}$ ) (Fig. 6C). Taken together, these results suggest that Gprk2 is not required for the constitutive dimerization of Smo through the Smo $\mathrm{N}$-terminal region but is required for Smo to adopt the active conformation in response to Hh stimulation.

Mutating the GPS sites to Ala in the SmoSD123 background (SDGPSA12) also reduced FRET $^{\mathrm{C}}$ but to a lesser extent as compared with Gprk2 RNAi (Fig. 6A). FRET between SmoSDGPSA12-CFP $/ \mathrm{YFP}^{\mathrm{C}}$ was further reduced by Gprk2 RNAi (Fig. 6A). In addition, FRET between SmoSDGPSD-CFP $/$ YFP $^{\mathrm{C}}$ was also reduced by Gprk2 RNAi (Fig. 6D). Thus, mutating the GPS sites did not abrogate the effect of Gprk2 RNAi on Smo conformation, suggesting that Gprk2 may regulate Smo conformation and C-terminal dimerization through a mechanism paralleled to phosphorylation at the GPS sites. To determine whether Gprk2 can promote the active Smo conformation independently of its kinase activity, we asked whether overexpression of Gprk $2^{\mathrm{KM}}$ could reverse the effect of Gprk2 RNAi on Smo FRET. As shown in Figure $6 \mathrm{D}$, transfection of either Flag-tagged wild-type Gprk2 (Fg-Gprk2) or Gprk2 ${ }^{\mathrm{KM}}$ (Fg-Gprk $\left.{ }^{\mathrm{KM}}\right)$ in cells treated with Gprk2 RNAi could rescue the FRET between. SmoSDGPSD12-CFP ${ }^{\mathrm{C}} / \mathrm{YFP}^{\mathrm{C}}$. In contrast, only Fg-Gprk2 but not Fg-Gprk2 ${ }^{\mathrm{KM}}$ reversed the effect of Gprk2 RNAi on the FRET between SmoSD-CFP $/ \mathrm{YFP}^{\mathrm{C}}$. Taken together, these results demonstrate that Gprk2 promotes the active Smo conformation through both phosphorylation-dependent and phosphorylation-independent mechanisms.

\section{Gprk2 interacts with Smo}

To explore the phosphorylation-independent mechanism by which Gprk2 promotes the active Smo conformation, we examined whether Gprk2 forms a complex with Smo. When expressed in S2 cells, Flag-tagged Gprk2 (Fg-Gprk2) coimmunoprecipitated with Myc-tagged full-length Smo (Myc-SmoFL) or Smo C-tail (Myc-SmoCT) but not with a truncated Smo lacking the C-tail (Myc-Smo $\Delta \mathrm{C}$ ) (Fig. 7AB), suggesting that Gprk2 interacts with Smo through its C-tail. Interaction between Smo and Gprk2 is independent of Gprk2 kinase activity, as Myc-Smo and FgGprk2 ${ }^{\mathrm{KM}}$ associated with each other in the coimmunoprecipitation assay (Supplemental Fig. S7). In addition, Smo interacted with mGRK5 (Supplemental Fig. S7), consistent with the observation that mGRK5 can functionally replace
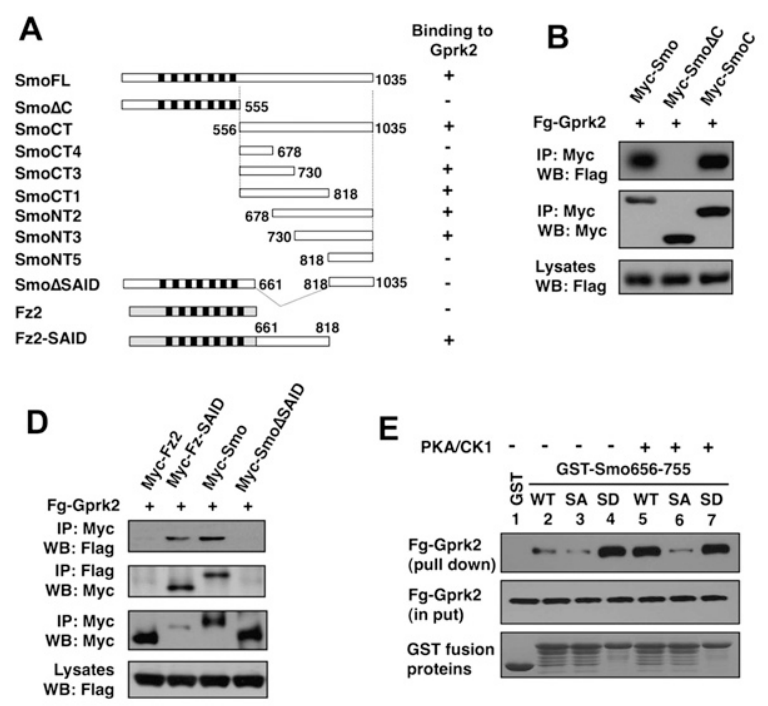

$\mathbf{F}$
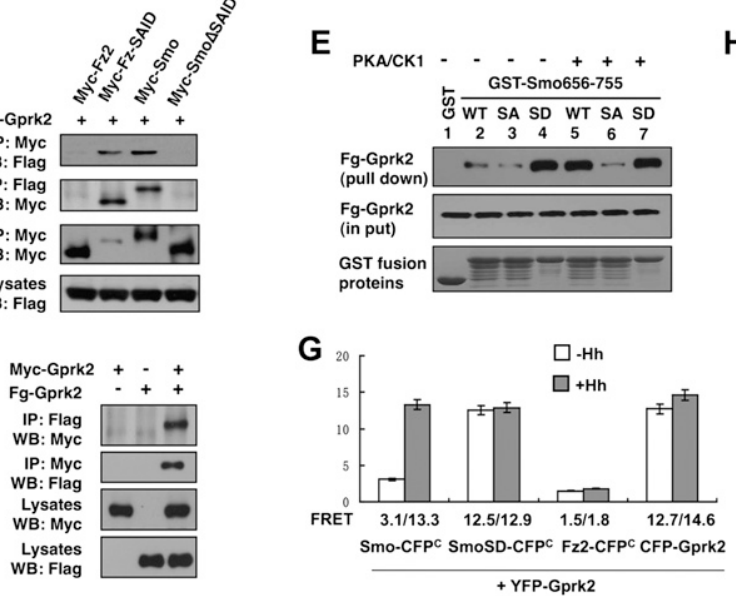

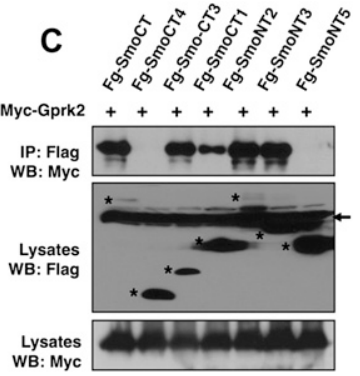

H

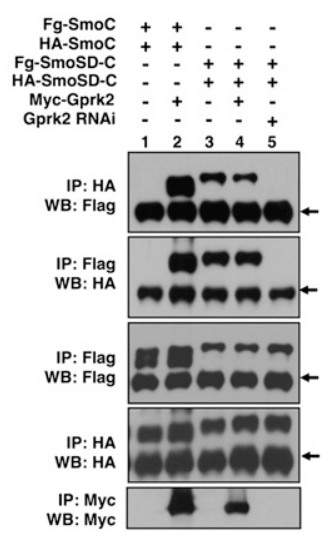

Figure 7. Gprk2 interacts with the SAID and promotes Smo C-tail dimerization. $(A)$ Schematic drawing of full-length Smo and its deletion mutants, Fz2, and Fz2-Smo chimeric protein (Fz2-SAID), in which the SAID is fused to the C terminus of Fz2. The ability of individual constructs to interact with Gprk2 is indicated on the right. $(B-D)$ Coimmunoprecipitation assays to determine Smo/Gprk2 interaction. S2 cells were transfected with indicated Myc-tagged or Flag (Fg)-tagged Smo or Fz2 and Gprk2 constructs, followed by immunoprecipitation and Western blot analysis with the indicated antibodies. Cell lysates were also directly immunoblotted by the indicated antibodies. Asterisks in $C$ indicate the positions of full-length or truncated Smo. The arrow in $C$ indicates IgG. (E) GST pull-down assay to determine Smo/Gprk2 interaction and its regulation by PKA/CK1mediated phosphorylation. GST-Smo fusion protein containing Smo656-755 with wildtype sequence (WT); PKA sites mutated to Ala (SA) or PKA/CK1 sites mutated to Asp (SD) were purified from bacteria, treated with $(+)$ or without $(-)$ PKA and CK1, and incubated with cell lysates derived from S2 cells expressing a Flag-tagged Gprk2 (Fg-Gprk2). Fg-Gprk2 bound to GST-Smo fusion proteins were pulled down by glutathione beads and detected by Western blot with anti-Flag antibody. (F) Self-association of Gprk2. S2 cells were transfected with Myc-tagged or Flag-tagged Gprk2 individually or in combination. Cell lysates were subjected to immunoprecipitation and Western blot analysis with the indicated antibodies. $(G)$ FRET analysis to determine Smo/Gprk2 interaction and Gprk2 self-association in intact cells. Numbers indicate the FRET efficiency from indicated CFP- and YFP-tagged constructs expressed in S2 cells. Mean \pm SD; $n \geq 20$. $(H)$ Gprk2 promotes dimerization of Smo C-tail. Flag- and HA-tagged wild-type Smo C-tail (SmoC) or its phosphomimetic form (SmoSD-C) were transfected into S2 cells with or without Myc-Gprk2, or into S2 cells treated with Gprk2 dsRNA (Gprk2 RNAi). Cell lysates were subjected to immunoprecipitation and Western blot analysis with the indicated antibodies. Arrows indicate IgG. 
Gprk2 in vivo (Fig. 2M). Interaction between Smo and Gprk2 is specific because Fg-Gprk2 did not pull down Myc-tagged Frizzled2 (Fz2) (Fig. 7D), which is the closest relative of Smo. Moreover, Smo did not interact with Drosophila Gprk1 using the same assay (Supplemental Fig. S7).

Further deletion analysis suggested that two nonoverlapping regions between amino acids 661 and 818 mediate Gprk2 binding (Fig. 7A,C). Deletion of amino acids 661-818 in the context of full-length Smo (SmosSAID) abolished Gprk2 binding, whereas fusion of amino acids 661-818 to Fz2 (Fz2-SAID) confers Gprk2 binding to the fusion protein (Fig. 7A,D). These observations demonstrate that Smo661-818, which contains the SAID, is both necessary and sufficient for Gprk2 binding.

To further characterize the Gprk2/Smo interaction and its regulation, we carried out a GST pull-down assay in which a GST fusion protein containing the SAID from amino acids 656 to 755 (GST-Smo656-755) was incubated with extracts from S2 cells expressing Fg-Gprk2. GSTSmo656-755 but not GST pulled down Fg-Gprk2 (Fig. 7E, lanes 1,2). Interestingly, PKA and CK1 phosphorylation of GST-Smo656-755 increased its binding affinity to FgGprk2 (Fig. 7E, lane 5), and mutating the three PKA sites to Ala (GST-Smo656-755SA) abolished this effect (Fig. $7 \mathrm{E}$, lane 6). On the other hand, mutating the PKA/CK1 sites to Asp (GST-Smo656-755SD) increased Fg-Gprk2 binding regardless of PKA/CK1 treatment (Fig. 7E, lanes $4,7)$. These results demonstrate that PKA/CK1-mediated phosphorylation of the SAID enhances its binding to Gprk2.

To confirm the Smo/Gprk2 interaction in intact cells, we applied FRET analysis. S2 cells were transfected with $\mathrm{N}$ terminally YFP-tagged Gprk2 (YFP-Gprk2) and Smo$\mathrm{CFP}^{\mathrm{C}}$, SmoSD-CFP ${ }^{\mathrm{C}}$, or Fz2-CFP ${ }^{\mathrm{C}}$; treated with or without Hh-conditioned medium; and subjected to FRET analysis. YFP-Gprk2 was tightly associated with the plasmic membrane (Supplemental Fig. S8), likely due to its lipid modification, as observed for mammalian GRK family kinases (Pitcher et al. 1998). YFP-Gprk2 was largely colocalized with $\mathrm{SmoSD}-\mathrm{CFP}^{\mathrm{C}}$ and $\mathrm{Fz} 2-\mathrm{CFP}^{\mathrm{C}}$ as well as $\mathrm{Smo}^{-\mathrm{CFP}}{ }^{\mathrm{C}}$ after Hh treatment (Supplemental Fig. S8). FRET between Smo-CFP ${ }^{\mathrm{C}}$ and YFP-Gprk2 was low in the absence of $\mathrm{Hh}$ $(3.1 \%)$ but increased dramatically after $\mathrm{Hh}$ treatment $(13.3 \%)$, whereas FRET between SmoSD-CFPC and YFPGprk2 was high regardless of Hh treatment (Fig. 7G). In contrast, there is no significant FRET between Fz2-CFP and YFP-Gprk2 (Fig. 7G). These observations suggest that $\mathrm{Hh}$ and PKA/CK1-mediated phosphorylation promotes Smo/Gprk2 association. In addition, the high FRET between Smo and Gprk2 suggests that they interact directly.

\section{Gprk2 forms a dimer/oligomer and promotes dimerization/oligomerization of Smo C-tail}

Interestingly, we also observed high FRET between CFPGprk2 and YFP-Gprk2 regardless of Hh treatment (Fig. 7G), suggesting that Gprk2 forms a constitutive dimer or oligomer. To further test whether Gprk2 can selfassociate, we expressed two differently tagged Gprk2s in
S2 cells and carried out immunoprecipitation assays. As shown in Figure 7F, Myc-tagged Gprk2 (Myc-Gprk2) pulled down Fg-Gprk2 and vice versa.

Since Gprk2 can self-associate, binding of Gprk2 to Smo C-tail may facilitate its dimerization/oligomerization, which could explain why Gprk2 is required for Hhand phosphorylation-induced proximity of Smo C-tails. To test this possibility, we transfected S2 cells with FlagSmo C-tail (Fg-SmoC) and HA-tagged Smo C-tail (HASmoC) in the absence or presence of Myc-Gprk2 cotransfection. In the absence of Myc-Gprk2, Fg-SmoC and HA-SmoC did not coimmunoprecipitate (Fig. 7H, lane 1); however, coexpression of Myc-Gprk2 allowed FgSmoC and HA-SmoC to associate with each other (Fig. $7 \mathrm{H}$, lane 2). In agreement with our previous findings that phosphorylation-mimetic mutation promotes Smo C-tail self-association (Zhao et al. 2007), Fg-SmoSD-C and HASmoSD-C coimmunoprecipitated regardless of exogenously expressed Gprk2 (Fig. 7H, lanes 3,4); however, this association was diminished by Gprk2 RNAi (Fig. 7H, lane 5), suggesting that endogenous Gprk2 is required for SmoSD-C self-association. Collectively, these observations suggest that PKA/CK1-mediated phosphorylation recruits Gprk2 to promote Smo C-tail dimerization/ oligomerization.

\section{Discussion}

In this study, we carried out a genetic modifier screen for novel $\mathrm{Hh}$ signaling components and identified Gprk2 as a positive regulator of Smo. Consistent with previous reports (Molnar et al. 2007; Cheng et al. 2010), we found that Gprk2 is required for high but not low levels of $\mathrm{Hh}$ signaling activity. We provided the first evidence that Gprk2 is a Smo kinase and that Gprk2 promotes maximal Smo activity by phosphorylating $\mathrm{S}_{741} / \mathrm{T}_{742}$ in Smo C-tail. Furthermore, we uncovered a kinase-independent function of Gprk2 in Hh signaling. We demonstrated that Gprk2 forms a dimer/oligomer and binds Smo C-tail to promote the active state of Smo. Thus, our study reveals a novel mechanism for regulating a GPCR-like protein by GRK.

\section{Gprk2 regulates th signaling by phosphorylating Smo}

Previous studies suggest that Drosophila Gprk2 and mammalian GRK2 are involved in Smo phosphorylation because their knockdown in cultured cells either increased Smo mobility on SDS-PAGE or decreased metabolic labeling of Smo by $\gamma^{-32}$ p-ATP (Chen et al. 2004; Cheng et al. 2010). However, these studies did not distinguish whether Gprk2/GRK2 phosphorylates Smo directly or indirectly through regulating other kinases. Neither did they reveal any biological relevance of Gprk2/GRK2mediated phosphorylation in $\mathrm{Hh}$ signaling, since the relevant phosphorylation sites on Smo were not identified. In an in vitro kinase assay using purified substrates and a recombinant GRK, we found that Smo is phosphorylated by GRK at $S_{741} / T_{742}$ and $S_{1013} / S_{1015}$. Our mutagenesis study demonstrated that phosphorylation at $\mathrm{S}_{741} / \mathrm{T}_{742}$ is required for optimal Smo activation. Indeed, a previous study showed that $S m o$ is phosphorylated at $S_{741} / T_{742}$ in 
cultured cells in the presence of Hh (Zhang et al. 2004). In further agreement with the functional significance of $\mathrm{S}_{741} /$ $\mathrm{T}_{742}$ phosphorylation, conserved $\mathrm{S} / \mathrm{T}$ residues are found at the corresponding location in other insect Smo proteins (FlyBase).

Interestingly, our in vitro kinase assay revealed that phosphorylation of $S_{741} / T_{742}$ by Gprk2 is regulated by PKA/CK1 phosphorylation at adjacent Ser residues, including $\mathrm{S}_{740}, \mathrm{~S}_{743}$, and $\mathrm{S}_{746}$. Previous studies in mammalian systems suggest that GRKs tend to phosphorylate S/ $\mathrm{T}$ residues embedded in an acidic environment (Premont et al. 1995). Phosphorylation at $\mathrm{S}_{740}, \mathrm{~S}_{743}$, and $\mathrm{S}_{746}$ improves the acidic environment for $S_{741} / T_{742}$, which may account for the observed stimulation of $S_{741} / T_{742}$ phosphorylation by PKA/CK1. Indeed, mutating $\mathrm{S}_{740}, \mathrm{~S}_{743}$, and $\mathrm{S}_{746}$ to Ala abolished PKA/CK1-mediated stimulation of $\mathrm{S}_{741} / \mathrm{T}_{742}$ phosphorylation, whereas converting these residues to acidic residues mimicked PKA/CK1-mediated stimulation. As Hh induces Smo phosphorylation by PKA and CK1, phosphorylation at $S_{741} / T_{742}$ by Gprk2 is likely to be stimulated by $\mathrm{Hh}$ in vivo.

\section{A kinase-independent role of Gprk2 in Hh signaling}

Although phosphomimetic mutation at $\mathrm{S}_{741} / \mathrm{T}_{742}$ promotes Smo activity, it does not bypass the requirement for Gprk2 for optimal Smo activation because SmoSDGPSD failed to induce ectopic en expression in Gprk2 mutant discs. This implies that Gprk2 promotes Hh signaling through a mechanism in parallel to $S_{741} / T_{742}$ phosphorylation. It is possible that Gprk2 might act at an additional step downstream from Smo activation by phosphorylating intracellular Hh signaling components, or at the level of Smo activation by phosphorylating Smo at additional sites that have been missed by our in vitro kinase assay. However, our finding that the constitutively active form of Smo lacking the SAID (Smo $\Delta 661-818)$ is insensitive to Gprk2 inactivation suggests that Gprk2 acts mainly at the level of Smo, although we cannot rule out the possibility that Gprk2 may also play a minor role downstream from Smo. Interestingly, we found that the kinase-dead form of Gprk2 (Gprk2 ${ }^{\mathrm{KM}}$ ) can rescue the activity defect of SmoSDGPSD in Gprk2 mutants, demonstrating that Gprk2 also regulates Smo in a phosphorylation-independent manner. The observation that Gprk2 ${ }^{\mathrm{KM}}$ does not rescue the activity defect of SmoSD123 in Gprk2 mutants suggests that the phosphorylation-dependent and phosphorylation-independent mechanisms act in parallel rather than redundantly to promote Smo activation. Furthermore, we obtained evidence that Gprk2 interacts with the SAID independently of its kinase activity. Therefore, we propose that Gprk2 promotes Smo activation by counteracting Smo autoinhibition through binding to and phosphorylating the SAID.

\section{Gprk2 regulates Smo level and conformation}

At least two paralleled mechanisms have been attributed to Smo activation by Hh: (1) Smo cell surface accumulation, and (2) conformation change in Smo C-tail (Jiang and Hui 2008). Intriguingly, we found that loss of Gprk2 resulted in increased rather than decreased Smo levels in cells that are not exposed to Hh or are exposed to low levels of Hh (Fig. 5A). Other groups made similar observations (Molnar et al. 2007; Cheng et al. 2010). However, unlike Hh stimulation, which preferentially stabilizes Smo on the cell surface, Gprk2 inactivation appears to stabilize Smo both inside the cell and on the cell surface (Fig. 5B). Furthermore, in the presence of high levels of $\mathrm{Hh}$ where Smo is accumulated at high levels on the cell surface, Gprk2 inactivation does not cause any discernible changes in either the level or subcellular distribution of Smo. Thus, the reduced Smo activity in Gprk2 mutant cells exposed to high levels of Hh is unlikely to be due to a change in Smo level or subcellular localization.

It is not clear what role Gprk2-mediated down-regulation of Smo levels might play in Hh signaling, although this may reflect an ancient mechanism by which GRK family kinases "desensitize" GPCRs. In this regard, Gprk2mediated down-regulation could serve as a mechanism to restrict the basal level of Hh signaling activity or to terminate or tune down Hh signaling activity once the Hh signal is withdrawn. However, this negative role of Gprk2 could be masked by its positive role. The mechanism by which Gprk2 down-regulates Smo levels remains unclear, although the kinase activity of Gprk2 appears to be required. Gprk2 could phosphorylate Smo and/or other proteins to promote Smo internalization and degradation. High levels of Hh could counteract Gprk2-mediated down-regulation of Smo by preventing Gprk2-meidated Smo internalization or by promoting Smo recycling.

Our FRET analysis provided strong evidence that Gprk2 is required for Smo to adopt and/or maintain its active conformation in response to Hh stimulation. Our previous study suggested that $\mathrm{Hh}$ induces a conformational switch in Smo C-tail that is mediated by PKA and CK1 phosphorylation (Zhao et al. 2007). In the absence of Hh, the Smo C-tail adopts a closed conformation in which the tail folds back, resulting in a close proximity between the $\mathrm{C}$ terminus and the third intracellular loop. The closed conformation is maintained, at least in part, through intramolecular electrostatic interactions between the multiple Arg clusters in the SAID and multiple acidic clusters near the $\mathrm{C}$ terminus. Hh-induced phosphorylation at PKA and CK1 sties disrupts the intramolecular electrostatic interactions, resulting in unfolding of the C-tail, which is reflected by a decreased intramolecular FRET (FRET ${ }^{\mathrm{L} 3}$ ). In addition, phosphorylation promotes dimerization of two C-tails within a Smo homodimer, leading to increased proximity of the two $\mathrm{C}$ termini, as reflected by an increased C-terminal FRET (FRET ${ }^{\mathrm{C}}$ ). Multiple intermediate conformational states may exist, depending on the levels of Smo phosphorylation, as increasing the number of phosphomimetic mutations progressively decreased FRET $^{\mathrm{L} 3 \mathrm{C}}$ and gradually increased FRET ${ }^{\mathrm{C}}$ (Zhao et al. 2007). We found that both an Hh-induced decrease in $\mathrm{FRET}^{\mathrm{L} 3 \mathrm{C}}$ and an Hhinduced increase in $\mathrm{FRET}^{\mathrm{C}}$ were compromised by loss of Gprk2 (Fig. 6), suggesting that Gprk2 is critical for Smo to adopt and/or maintain the fully open conformation.

How does Gprk2 regulate Smo conformation? Our genetic and FRET analyses demonstrated that Gprk2 
promotes high levels of Hh signaling activity and regulates Smo conformation through both phosphorylationdependent and phosphorylation-independent mechanisms. Furthermore, we found that Gprk2 self-associates, binds the SAID, and promotes self-association of Smo C-tail. Interestingly, both Gprk2/SAID interaction and $\mathrm{S}_{741} / \mathrm{T}_{742}$ phosphorylation by Gprk2 are enhanced by PKA/CK1 phosphorylation. Taken together, we propose the following model to account for the regulation of Smo conformation by Gprk2 (Fig. 8). In response to Hh, PKA/ CK1-mediated phosphorylation of Smo C-tail promotes its unfolding and dimerization; however, in the absence of Gprk2, the open conformational state of Smo is unstable and may exist in equilibrium with the closed and/ or partially open conformational states. Phosphorylation of Smo by PKA/CK1 promotes the binding of Gprk2 to the SAID and phosphorylation at $\mathrm{S}_{741} / \mathrm{T}_{742}$, both of which may stabilize Smo in the fully open and active conformation by preventing refolding of Smo C-tail and by "cross-linking" the two C-tails within a Smo dimer via dimerization of Gprk2. In essence, Gprk2 may function as a "molecular clamp" to promote the clustering of Smo C-tails. It is also possible that Gprk2 could cross-link two or more Smo dimers to form high-order oligomers, which might be essential for high levels of Hh signaling activity. Our study thus reveals an unanticipated complexity in the regulation of Smo conformational states, and provides the first evidence that Smo conformation states are regulated by not only phosphorylation and intramolecular interactions, but also intermolecular interactions. It is possible that the closed conformation state of Smo is also regulated by intermolecular interactions in addition to intramolecular interactions. For example, it has been shown that Fu can directly bind the Smo $\mathrm{C}$ terminus in the absence of Hh (Malpel et al. 2007), and this interaction may help stabilize the closed conformation of Smo $\mathrm{C}$-tail. Indeed, disrupting $\mathrm{Smo} / \mathrm{Fu}$ interaction led to increased basal activity of Smo (Malpel et al. 2007).

\section{Implication on mammalian Smo regulation}

Recent studies have emphasized the differences between vertebrate and Drosophila $\mathrm{Hh}$ signaling mechanisms.

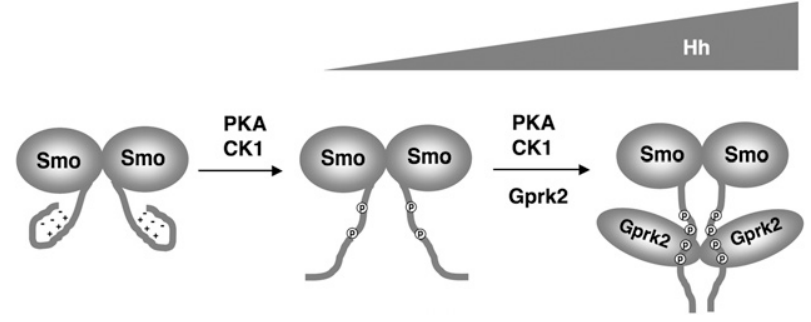

Figure 8. Model for regulating Smo activation state by Gprk2. Hh-induced phosphorylation by PKA and CK1 leads to unfolding of Smo C-tail and promotes its association with and phosphorylation by Gprk2. Both Gprk2 binding and phosphorylation stabilize Smo in the active conformation by preventing refolding. In addition, Gprk2 forms a dimer/oligomer and promotes the active state of Smo by cross-linking Smo C-tails.
The sequence divergence between Drosophila and vertebrate Smo proteins and the lack of conserved PKA/CK1 phosphorylation sites in vertebrate Smo proteins have led to the proposal that vertebrate Smo proteins are activated through fundamentally distinct mechanisms (Huangfu and Anderson 2006; Varjosalo et al. 2006). Nevertheless, our previous study revealed that Shh induces a conformational change in mSmo similar to that of $\mathrm{dSmo}$, and forced clustering of $\mathrm{mSmo}$ also leads to pathway activation (Zhao et al. 2007). GRK2 has been implicated as a positive regulator of the Shh pathway both in cultured cells and in vivo (Chen et al. 2004; Meloni et al. 2006; Philipp et al. 2008), and mSmo phosphorylation is affected by GRK2 silencing, although direct phosphorylation of mSmo by GRK2 has not been demonstrated. It is possible that GRK2 may substitute the role of PKA and CK1 and act as a major Smo kinase in vertebrates to promote the active Smo conformation. Alternatively, GRK2 may act in conjunction with other GRKs and/or yet-tobe-identified kinases to regulate Smo conformation, subcellular localization, and activity in vertebrates. The relatively weak phenotypes exhibited by GRK2 mutants are consistent with the latter possibility (Philipp et al. 2008). Our study also raised an interesting possibility that GRK2 may regulate mSmo not only by phosphorylation, but also by a kinase-independent mechanism such as a protein-protein interaction. Further investigation of the mechanism by which GRK2 and other kinases regulate mSmo will shed an important light on how vertebrate Smo activation is achieved.

\section{Materials and methods}

\section{Mutations and transgenes}

UAS-Smo ${ }^{-P K A 12}$ has been described (Jia et al. 2004). The Gal4 drivers MS1096 and C765 were described in FlyBase. The genetic modifier screen was carried out by crossing females of the genotype MS1096; UAS-Smo-PKA12/TM2 or C765, UAS$S o^{-P K A 12} / T M 6 B$ with males of Exilexis deficiency lines obtained from the Bloomington Stock Center. The F1 progenies were scored for any modifications of the fused wing phenotype. Gprk2 ${ }^{06936}$ has been described (Schneider and Spradling 1997). Excision alleles of Gprk2 were generated by crossing Gprk $2^{06936}$ with $\Delta 2-3$ flies that carry the transposase. Progenies that lost $r y^{+}$ were crossed with $C 765$, UAS-Smo ${ }^{-P K A 12} / T M 6 B$. Precise excision alleles no longer modified the fused wing phenotype, whereas several imprecise excision lines, including Gprk $2^{\Delta 15}$, enhanced the wing phenotype similarly to the Gprk2 deficiency line $D f(3 R)$ Exel6219. Gprk2 ${ }^{\Delta 15}$ mutant clones were generated in yw 122; FRT82 Gprk2 ${ }^{\Delta 15} / F R T 82$ hs-Myc-GFP.

To generate Flag- or Myc-tagged Gprk2 constructs, the Gprk2 coding sequence was amplified by PCR and inserted in-frame in the pUAST-Flag or pUAST-Myc vector (Chao and Jiang 2007). To construct the kinase-dead form of Gprk2, the conserved lysines K338 and K339 within the kinase catalytic domain of Gprk2 were mutated into Met using PCR-based site-directed mutagenesis. To construct UAS-Gprk2-CFP $/ \mathrm{YFP}^{N}, \mathrm{CFP} / \mathrm{YFP}$ was inserted in-frame before the Gprk2 start codon. Amino acid substitutions of GPS1 and GPS2 were generated by PCR-based sitedirected mutagenesis. To construct UAS-SmoGPSA-CFP/YFP and UAS-SmOSDGPSD-CFP/YFP, CFP/YFP was inserted inframe after the $\mathrm{C}$ termini of the corresponding Smo variants. 
To construct UAS-GPRK2-RNAi, a genomic DNA fragment encoding Gprk2 amino acids 180-385 was amplified by PCR and cloned between the BglII and XhoI sites of the pUAST vector, with the corresponding cDNA fragment inserted in a reverse orientation between the XhoI and XbaI sites. The PhiC31 integration system was used to integrate Smo transgenes into the 75B1 attP locus (Bischof et al. 2007; Jia et al. 2009). To generate GST-Smo fusion constructs, DNA fragments encoding Smo C-terminal regions with either the wild-type sequence or point mutations in phosphorylation sites were amplified by PCR and inserted between BamHI and NotI sites in the $p G E X-4 T-2$ vector.

Cell culture, transfection, immunoprecipitation, GST pull-down, Western blot, and immunostaining

Drosophila S2 cells were cultured in Drosophila SFM (Invitrogen) with $10 \%$ fetal bovine serum (FBS), $100 \mathrm{U} / \mathrm{mL}$ penicillin, and $100 \mathrm{mg} / \mathrm{mL}$ streptomycin at $23^{\circ} \mathrm{C}$. Transfection was carried out by Calcium Phosphate Transfection Kit (Specialty Media) according to manufacturer's instructions. Hh-conditioned medium treatment was carried out as described (Lum et al. 2003). Briefly, Hh-N stably expressed S2 cells were selected in $200 \mu \mathrm{g} / \mathrm{mL}$ hygromycine. Hh-conditioned medium was prepared by culturing cells without hygromycine but with $0.7 \mathrm{mM} \mathrm{CuSO}_{4}$ for $1 \mathrm{~d}$. The medium was harvested and sterilized by filtration. Unless mentioned otherwise, Hh-conditioned medium was used at a 6:4 dilution ratio by fresh medium. Immunoprecipitation and Western blot analysis were carried out using standard protocols as described previously (Zhang et al. 2005). For GST pull-down assay, GST fusion proteins bound to the glutathione beads were washed three times with ice-cold PBS containing $1 \%$ NP-40, and were incubated with cell lysates from S2 cells expressing Flag-tagged Gprk2 proteins for $1 \mathrm{~h}$ at $4^{\circ} \mathrm{C}$ with occasional mixing. The beads were washed again five times with PBS plus $1 \%$ NP-40 before separation on SDS-PAGE, followed by Western blot using anti-Flag antibody. For the Smo cell surface staining assay, S2 cells were harvested and washed with PBS, fixed with $4 \%$ formaldehyde at room temperature for $20 \mathrm{~min}$, and incubated with the mouse anti-SmoN antibody in PBS at room temperature for $30 \mathrm{~min}$. Cells were washed three times by PBS followed by secondary antibody staining. Immunostaining of imaginal discs was carried out as described (Jiang and Struhl 1995). Antibodies used in this study were mouse anti-En (Developmental Studies Hybridoma Bank [DSHB]), mouse anti-Ptc (DSHB), mouse anti-SmoN (DSHB), rabbit and mouse anti-Flag (Sigma), mouse anti-Myc (Santa Cruz Biotechnology), mouse anti-HA (Santa Cruz Biotechnology), mouse anti-Ci 2A1 (Motzny and Holmgren 1995), mouse anti-GFP (Millipore), rabbit anti-GFP (Santa Cruz Biotechnology), and guinea pig anti-dGprk2 (Cheng et al. 2010).

\section{In vitro kinase assay}

In vitro kinase assay using recombinant GRK5 was performed according to the manufacturer's instructions (Upstate Biotechnologies, 14-714). Gprk2 in vitro kinase reaction was performed for $1 \mathrm{~h}$ at $30^{\circ} \mathrm{C}$ in kinase buffer $(20 \mathrm{mM}$ Tris- $\mathrm{HCl}$ at $\mathrm{pH} 8.0,2 \mathrm{mM}$ EDTA, $10 \mathrm{mM} \mathrm{MgCl} 2,1 \mathrm{mM}$ DTT), with Fg-Gprk2 or FgGprk2 ${ }^{\mathrm{KM}}$ immunoprecipitated from S2 cells transfected with Fg-Gprk2 or Flag-Gprk ${ }^{\mathrm{KM}}$ expression construct, GST fusion proteins, and $0.1 \mathrm{mM}$ ATP containing $10 \mathrm{mCi}$ of $\gamma^{-}{ }^{32} \mathrm{p}$-ATP. PKA and CK1 were purchased from New England Biolabs. After the GST fusion proteins were incubated with PKA and CK1 in the presence of cold ATP for $30 \mathrm{~min}$ at $30^{\circ} \mathrm{C}$, the reactions were stopped by adding $10 \mathrm{mM}$ D4476 and H-89 /CK1 and PKA inhibitors, respectively). PKA/CK1-treated GST fusion proteins were further processed with GRK5 or Gprk2 phosphorylation for
$1 \mathrm{~h}$ at $30^{\circ} \mathrm{C}$ in the presence of $\gamma_{-}{ }^{32} \mathrm{p}$-ATP. The reactions were finally stopped by adding $4 \times$ SDS loading buffer and were boiled for $5 \mathrm{~min}$ at $100^{\circ} \mathrm{C}$. Phosphorylation of GST fusion proteins was analyzed by autoradiography after SDS-PAGE.

\section{RNAi in Drosophila S2 cells}

dsRNA was generated by MEGAscript High-Yield Transcription Kit (Ambion, \#AM1334) according to the manufacturer's instructions. A DNA template targeting Gprk2 amino acids 124290 was generated by PCR and used for making Gprk2 dsRNA. dsRNA targeting the Firefly Luciferase coding sequence was used as a control. For the RNAi knockdown experiments described in Figure 6, S2 cells were cultured in serum-free SFM medium containing the indicated dsRNA for $8 \mathrm{~h}$ at $25^{\circ} \mathrm{C}$. After adding FBS to a final concentration of $10 \%$, dsRNA-treated cells were cultured overnight before transfection with CFP/YFPtagged Smo constructs. After additional culturing for $2 \sim 3 \mathrm{~d}$, the cells were collected for FRET assay. For Gprk2 rescue experiments in cultured S2 cells, $2 \mathrm{~d}$ after transfection of CFP/YFP-tagged Smo constructs, the cells were changed into fresh SFM medium containing $10 \%$ FBS, transfected with Gprk2 or Gprk2 ${ }^{\mathrm{KM}}$ expression construct, and subjected to FRET assay after culturing for two additional days.

\section{FRET analysis using confocal microscopy}

FRET analysis was carried out as described previously (Zhao et al. 2007). CFP- and YFP-tagged constructs in the $p U A S T$ vector were cotransfected into S2 cells together with an $u b$-Gal4 expression vector. Cells were washed with PBS, fixed with $4 \%$ formaldehyde for $20 \mathrm{~min}$, and mounted on slides in $80 \%$ glycerol. CFP signals were acquired with the $100 \times$ objective of a Zeiss LSM510 confocal microscope before (BP) and after (AP) photobleaching YFP. Each data set was calculated using 15-20 individual cells. In each cell, four or five regions of interest in a photobleached area were selected for analysis. The intensities of CFP signals were quantified by ImageJ software. The FRET efficiency was calculated using the formula FRET $\%=\left[\left(\mathrm{CFP}_{\mathrm{AP}}-\right.\right.$ $\left.\left.\mathrm{CFP}_{\mathrm{BP}}\right) / \mathrm{CFP}_{\mathrm{AP}}\right] \times 100$.

\section{Acknowledgments}

We thank Drs. Jeffrey Benovic, Robert Holmgren, and David Hipfner; the Bloomington Stock Center, and DSHB for reagents. This work was supported by grants from NIH, Welch Foundation (I-1603), and CPRIT (to J.J.). J.J. is a Eugene McDermott Endowed Scholar in Biomedical Science at UTSW.

\section{References}

Apionishev S, Katanayeva NM, Marks SA, Kalderon D, Tomlinson A. 2005. Drosophila Smoothened phosphorylation sites essential for Hedgehog signal transduction. Nat Cell Biol 7: 8692.

Aza-Blanc P, Ramirez-Weber F, Laget M, Schwartz C, Kornberg T. 1997. Proteolysis that is inhibited by Hedgehog targets Cubitus interruptus protein to the nucleus and converts it to a repressor. Cell 89: 1043-1053.

Bischof J, Maeda RK, Hediger M, Karch F, Basler K. 2007. An optimized transgenesis system for Drosophila using germline-specific phiC31 integrases. Proc Natl Acad Sci 104: 3312-3317.

Casali A, Struhl G. 2004. Reading the Hedgehog morphogen gradient by measuring the ratio of bound to unbound Patched protein. Nature 431: 76-80. 
Chao T, Jiang J. 2007. Using immunoprecipitation to study protein-protein interaction in the Hedgehog signaling pathway. Methods Mol Biol 397: 215-229.

Chen Y, Struhl G. 1996. Dual roles for patched in sequestering and transducing Hedgehog. Cell 87: 553-563.

Chen W, Ren XR, Nelson CD, Barak LS, Chen JK, Beachy PA, de Sauvage F, Lefkowitz RJ. 2004. Activity-dependent internalization of smoothened mediated by $\beta$-arrestin 2 and GRK2. Science 306: 2257-2260.

Cheng S, Maier D, Neubueser D, Hipfner DR. 2010. Regulation of smoothened by DROSOPHILA G-protein-coupled receptor kinases. Dev Biol 337: 99-109.

Denef N, Neubuser D, Perez L, Cohen SM. 2000. Hedgehog induces opposite changes in turnover and subcellular localization of patched and smoothened. Cell 102: 521-531.

Huangfu D, Anderson KV. 2006. Signaling from Smo to Ci/Gli: Conservation and divergence of Hedgehog pathways from Drosophila to vertebrates. Development 133: 3-14.

Ingham PW, McMahon AP. 2001. Hedgehog signaling in animal development: Paradigms and principles. Genes Dev 15: 3059-3087.

Jia J, Amanai K, Wang G, Tang J, Wang B, Jiang J. 2002. Shaggy/ GSK3 antagonizes Hedgehog signalling by regulating Cubitus interruptus. Nature 416: 548-552.

Jia J, Tong C, Wang B, Luo L, Jiang J. 2004. Hedgehog signalling activity of Smoothened requires phosphorylation by protein kinase A and Casein Kinase I. Nature 432: 1045-1050.

Jia J, Zhang L, Zhang Q, Tong C, Wang B, Hou F, Amanai K, Jiang J. 2005. Phosphorylation by double-time/CKI $\varepsilon$ and CKI $\alpha$ targets cubitus interruptus for Slimb/ $\beta$-TRCP-mediated proteolytic processing. Dev Cell 9: 819-830.

Jia H, Liu Y, Yan W, Jia J. 2009. PP4 and PP2A regulate Hedgehog signaling by controlling Smo and Ci phosphorylation. Development 136: 307-316.

Jiang J, Hui CC. 2008. Hedgehog signaling in development and cancer. Dev Cell 15: 801-812.

Jiang J, Struhl G. 1995. Protein kinase A and Hedgehog signalling in Drosophila limb development. Cell 80: 563-572.

Lum L, Yao S, Mozer B, Rovescalli A, Von Kessler D, Nirenberg M, Beachy PA. 2003. Identification of Hedgehog pathway components by RNAi in Drosophila cultured cells. Science 299: 2039-2045.

Malpel S, Claret S, Sanial M, Brigui A, Piolot T, Daviet L, Martin-Lanneree S, Plessis A. 2007. The last 59 amino acids of Smoothened cytoplasmic tail directly bind the protein kinase Fused and negatively regulate the Hedgehog pathway. Dev Biol 303: 121-133.

Meloni AR, Fralish GB, Kelly P, Salahpour A, Chen JK, WechslerReya RJ, Lefkowitz RJ, Caron MG. 2006. Smoothened signal transduction is promoted by $\mathrm{G}$ protein-coupled receptor kinase 2. Mol Cell Biol 26: 7550-7560.

Methot N, Basler K. 1999. Hedgehog controls limb development by regulating the activities of distinct transcriptional activator and repressor forms of Cubitus interruptus. Cell 96: 819831.

Methot N, Basler K. 2000. Suppressor of Fused opposes Hedgehog signal transduction by impeding nuclear accumulation of the activator form of Cubitus interruptus. Development 127: 4001-4010.

Molnar C, Holguin H, Mayor F Jr, Ruiz-Gomez A, de Celis JF. 2007. The G protein-coupled receptor regulatory kinase GPRK2 participates in Hedgehog signaling in Drosophila. Proc Natl Acad Sci 104: 7963-7968.

Motzny CK, Holmgren R. 1995. The Drosophila cubitus interruptus protein and its role in the wingless and hedgehog signal transduction pathways. Mech Dev 52: 137-150.
Ogden SK, Fei DL, Schilling NS, Ahmed YF, Hwa J, Robbins DJ. 2008. G protein $\mathrm{G} \alpha$ (i) functions immediately downstream of Smoothened in Hedgehog signalling. Nature. 456: 967-970.

Ohlmeyer JT, Kalderon D. 1998. Hedgehog stimulates maturation of Cubitus interruptus into a labile transcriptional activator. Nature 396: 749-753.

Philipp M, Fralish GB, Meloni AR, Chen W, MacInnes AW, Barak LS, Caron MG. 2008. Smoothened signaling in vertebrates is facilitated by a $\mathrm{G}$ protein-coupled receptor kinase. Mol Biol Cell 19: 5478-5489.

Pitcher JA, Freedman NJ, Lefkowitz RJ. 1998. G protein-coupled receptor kinases. Annu Rev Biochem 67: 653-692.

Premont RT, Inglese J, Lefkowitz RJ. 1995. Protein kinases that phosphorylate activated $\mathrm{G}$ protein-coupled receptors. FASEB J 9: 175-182.

Price MA, Kalderon D. 2002. Proteolysis of the Hedgehog signaling effector Cubitus interruptus requires phosphorylation by glycogen synthase kinase 3 and Casein Kinase 1. Cell 108: 823-835.

Robbins DJ, Nybakken KE, Kobayashi R, Sisson JC, Bishop JM, Therond PP. 1997. Hedgehog elicits signal transduction by means of a large complex containing the kinesin-related protein costal2. Cell 90: 225-234.

Sallese M, Mariggio S, D’Urbano E, Iacovelli L, De Blasi A. 2000. Selective regulation of $\mathrm{Gq}$ signaling by $\mathrm{G}$ protein-coupled receptor kinase 2: Direct interaction of kinase $\mathrm{N}$ terminus with activated goq. Mol Pharmacol 57: 826-831.

Schneider LE, Spradling AC. 1997. The Drosophila G-proteincoupled receptor kinase homologue Gprk2 is required for egg morphogenesis. Development 124: 2591-2602.

Sisson BE, Ziegenhorn SL, Holmgren RA. 2006. Regulation of Ci and $\mathrm{Su}(\mathrm{fu})$ nuclear import in Drosophila. Dev Biol 294: 258270.

Smelkinson MG, Zhou Q, Kalderon D. 2007. Regulation of CiSCFSlimb binding, Ci proteolysis, and hedgehog pathway activity by Ci phosphorylation. Dev Cell 13: 481-495.

Stone DM, Hynes M, Armanini M, Swanson TA, Gu Q, Johnson RL, Scott MP, Pennica D, Goddard A, Phillips H, et al. 1996. The tumour-suppressor gene patched encodes a candidate receptor for Sonic hedgehog. Nature 384: 129-134.

Strigini M, Cohen SM. 1997. A Hedgehog activity gradient contributes to AP axial patterning of the Drosophila wing. Development 124: 4697-4705.

Taipale J, Beachy PA. 2001. The Hedgehog and Wnt signalling pathways in cancer. Nature 411: 349-354.

Taipale J, Cooper MK, Maiti T, Beachy PA. 2002. Patched acts catalytically to suppress the activity of Smoothened. Nature 418: 892-897.

Varjosalo M, Taipale J. 2008. Hedgehog: Functions and mechanisms. Genes Dev 22: 2454-2472.

Varjosalo M, Li SP, Taipale J. 2006. Divergence of hedgehog signal transduction mechanism between Drosophila and mammals. Dev Cell 10: 177-186.

Villavicencio EH, Walterhouse DO, Iannaccone PM. 2000. The sonic hedgehog-patched-gli pathway in human development and disease. Am J Hum Genet 67: 1047-1054.

Wang G, Jiang J. 2004. Multiple Cos2/Ci interactions regulate Ci subcellular localization through microtubule dependent and independent mechanisms. Dev Biol 268: 493-505.

Wang QT, Holmgren RA. 2000. Nuclear import of cubitus interruptus is regulated by hedgehog via a mechanism distinct from Ci stabilization and Ci activation. Development 127: 3131-3139.

Wang G, Amanai K, Wang B, Jiang J. 2000. Interactions with Costal2 and suppressor of fused regulate nuclear translocation and activity of cubitus interruptus. Genes Dev 14: 2893-2905. 
Zhang C, Williams EH, Guo Y, Lum L, Beachy PA. 2004. Extensive phosphorylation of Smoothened in Hedgehog pathway activation. Proc Natl Acad Sci 101: 17900-17907.

Zhang W, Zhao Y, Tong C, Wang G, Wang B, Jia J, Jiang J. 2005. Hedgehog-regulated costal2-kinase complexes control phosphorylation and proteolytic processing of cubitus interruptus. Dev Cell 8: 267-278.

Zhao Y, Tong C, Jiang J. 2007. Hedgehog regulates smoothened activity by inducing a conformational switch. Nature 450: 252-258.

Zheng X, Mann RK, Sever N, Beachy PA. 2010. Genetic and biochemical definition of the Hedgehog receptor. Genes Dev 24: 57-71.

Zhu AJ, Zheng L, Suyama K, Scott MP. 2003. Altered localization of Drosophila Smoothened protein activates Hedgehog signal transduction. Genes Dev 17: 1240-1252. 


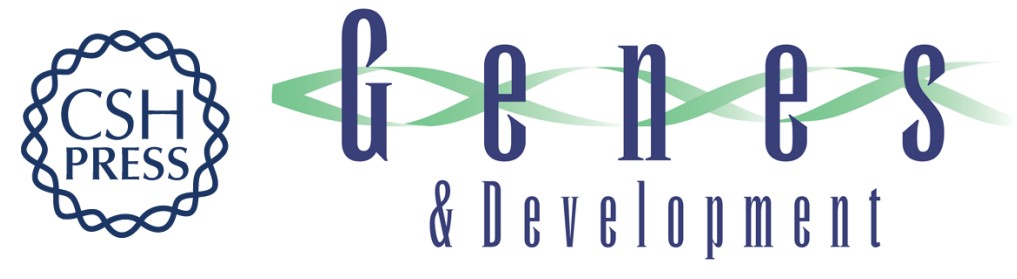

\section{G protein-coupled receptor kinase 2 promotes high-level Hedgehog signaling by regulating the active state of Smo through kinase-dependent and kinase-independent mechanisms in Drosophila}

Yongbin Chen, Shuang Li, Chao Tong, et al.

Genes Dev. 2010, 24:

Access the most recent version at doi:10.1101/gad.1948710

Supplemental http://genesdev.cshlp.org/content/suppl/2010/09/10/24.18.2054.DC1

Material

References This article cites 50 articles, 19 of which can be accessed free at:

http://genesdev.cshlp.org/content/24/18/2054.full.html\#ref-list-1

License

Email Alerting Receive free email alerts when new articles cite this article - sign up in the box at the top

Service right corner of the article or click here.

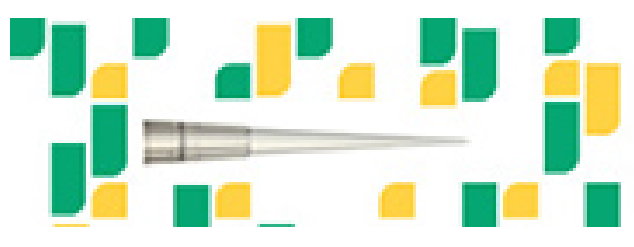

Focused on your science. 